\title{
A Novel Nonlinear Optimal Control Approach for the Dynamic Process of a Hybrid Electric Vehicle Equipped with Electromechanical Transmission
}

\author{
Changle Xiang, ${ }^{1}$ Donghao Zhang, ${ }^{2}$ Hailiang Zheng, ${ }^{1}$ Lijin Han, ${ }^{1}$ and Kun Huang ${ }^{1}$ \\ ${ }^{1}$ School of Mechanical Engineering, Beijing Institute of Technology, Beijing 100081, China \\ ${ }^{2}$ Department of Automotive Engineering, Tsinghua University, Beijing 100084, China
}

Correspondence should be addressed to Donghao Zhang; zhdonghao@tsinghua.edu.cn

Received 18 December 2014; Revised 10 May 2015; Accepted 11 May 2015

Academic Editor: Asier Ibeas

Copyright (C) 2015 Changle Xiang et al. This is an open access article distributed under the Creative Commons Attribution License, which permits unrestricted use, distribution, and reproduction in any medium, provided the original work is properly cited.

\begin{abstract}
Coordinatively controlling the engine and several motor/generators (MGs) during a dynamic process is a challenging problem because they are coupled together by the electromechanical transmission (EMT) system and all of them have strong nonlinear characteristics. We develop a novel nonlinear optimal control approach based on the multiobjective dynamic optimization model of the hybrid electric vehicle (HEV), which is equipped with an EMT system. In this approach, the current states of the components are obtained by using the state observation algorithm based on Kalman filtering; the future states of the components and the feasible region of the control variables are estimated by using the dynamic prediction algorithm based on the nonlinear model of the EMT system. Then, the control variables are achieved by using the optimal decision algorithm based on the hierarchical optimization and nonlinear programming, and the influence of the model error and the external disturbance are modified by using the feedback compensation algorithm. The simulation results illustrate the efficiency of the proposed control approach, and the test results verify its real-time performance.
\end{abstract}

\section{Introduction}

Hybrid electric vehicles (HEVs) have received much attention from researchers, governments, and manufacturers because of their high fuel economy and low emissions. The driving performance, which is especially significant for the heavyduty vehicles such as SUVs and trucks, can also be obviously improved due to the additional power of the battery. Additionally, the hybrid powertrain system can provide electricity to various electric appliances inside the vehicles. However, the overall performance of the HEVs strongly depends on the control strategies because there are several degrees of freedom in the powertrain system.

Existing control strategies of the HEVs were divided into two groups according to the mathematical description: the rule-based and the optimization-based methods $[1,2]$. These strategies were described as deterministic or fuzzy rules depending on engineers' experiences and heuristics. As they are easy to be mastered and suitable for real-time control, these strategies have been widely used and developed in the early stages. However, the potential performance of the HEVs can hardly be fully explored based only on engineers' intuition [3], resulting in the gradual emergence of various optimization-based strategies. Dynamic programming (DP) and equivalent consumption minimization strategy (ECMS) are the hottest optimization-based strategies [4-8]. The DP approach can obtain the globally optimal trajectories based on the driving cycle given in advance, but the solutions are not suitable for the other driving cycles. Therefore, it is noncausal and cannot be applied in real-time control process. The DP solutions were mainly used as a benchmark for the best performance and to optimize the parameters of the other control approaches [9-12]. The ECMS approach is an instantaneous optimization method, which can be applied on-line as a closed-loop controller [13, 14]. The equivalent factor is the core of this approach and is also dependent on the driving cycles. The ECMS was divided into four categories in [15], and the adaptive equivalent consumption 
minimization strategy (A-ECMS), which represents the latest ECMS approach, has the greatest potential of achieving optimal control during on-line operations [16].

To overcome the problem of the DP approach being dependent on the driving cycles, some researchers utilized the stochastic process to describe the possible vehicle speeds and proposed the stochastic dynamic programming (SDP) approach $[17,18]$. However, this approach needs the Markov model, which is built on the basis of a large number of driving cycles. Actually, the Markov model can hardly be established [19]. The model predictive control (MPC), which has been widely used in industrial fields, was introduced into the HEV s' control field and has become a research focus in recent years [20-24]. The MPC approach mainly consists of three parts: the predictive model, the receding horizon optimization, and the feedback compensation. The predictive model can be the model of SOC, the states of the engine and the MGs, or the driving cycles [21]. The rolling optimization algorithm can be the maximum principle, the quadratic programming, or the DP approach [22-24]. Thus, the MPC approach has become a general model-based control method, which can be divided into different types according to the prediction models and the optimization algorithms. For instance, the SDP approach can be considered as a kind of MPC algorithm, which takes the Markov model as the prediction model and the DP approach as the optimization algorithm.

To reduce the calculation time of the optimal control algorithms, some researchers have made significant attempts at different fields, such as robust control [25], intelligent control [26], game theory [27], and analytic and other methods $[28,29]$. These studies provide basis for the real-time implementation of the optimal control.

Electromechanical transmission (EMT), a kind of continuous variable transmission, can adjust the engine's speed to the optimal region using two or more motors [30]. Compared with the general hybrid system, the EMT system has some noticeable characteristics. For instance, the power level of the battery pack in the EMT system is much lower than that of the engine, so the battery pack is not used to provide energy for the vehicle but to compensate the power deviation between the MGs and the electric appliances in the dynamic process. The drivability and fuel economy are improved mainly with the MGs adjusting the engines' working points, and the current supply capacity is satisfied by the MGs by converting the engine's mechanical power to electricity. Therefore, the coordination control of the engine and the MGs is the core in the EMT system, which is a dynamic optimization problem.

Based on the nonlinear characteristics of the power components and their strong coupling interaction, the dynamic optimization model was built, and an innovative nonlinear optimal control approach was proposed in this paper. The control approach was named as OPDC because it consisted of four parts: state observation, dynamic prediction, optimal decision, and feedback compensation. The basic idea can be described as follows. Firstly, the current states of the system are obtained through the state observation algorithm. Secondly, the future states of the system are predicted, and the feasible region of the control variables is obtained through the dynamic prediction algorithm. Then the optimal control

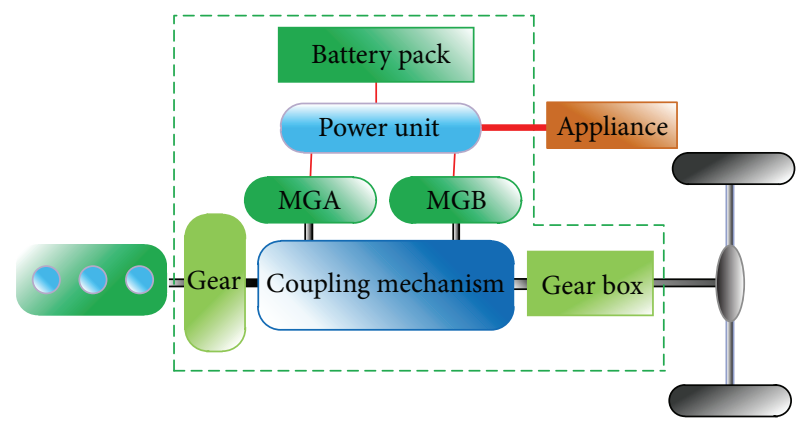

Figure 1: Structure diagram of the EMT system.

variables are derived through the optimal decision algorithm by utilizing the current and future states of the system. Lastly, the real-time modifications of the model parameters and the control parameters are done through the feedback compensation algorithm by utilizing the state deviation derived from the feedback. As it comprehensively uses the current and future information, the OPDC approach can fully explore the potential of the system. Moreover, the OPDC approach can be considered as a nonlinear model prediction control algorithm because it is a model-based control method and is suitable for the nonlinear system.

This paper is organized as follows. The principle of the EMT system is first introduced, and the nonlinear characteristics of the power components and their coupling interactions are analyzed in Section 2. The dynamic optimization model of the system is constructed in Section 3 based on the multiple optimization objectives and the nonlinear timevarying constraints. In Section 4, the OPDC approach is derived and applied in the EMT system. In Section 5, the simulation models of the HEV and the control unit are built, and the on-line test of the OPDC approach is carried out by using the dSPACE platform, which verifies real-time performance and effectiveness. Finally, concluding remarks are presented in Section 6.

\section{Characteristics of the EMT System}

2.1. Principle of the EMT System. As shown inside the dottedline frame in Figure 1, the EMT system consists of not only mechanical transmission parts, such as the front gears, the coupling mechanism, and the rear gearbox, but also the electrical transmission parts such as the MGs (MGA and MGB), the power unit, and the battery pack. The coupling mechanism consists of several planetary, clutches, and brakes, as shown in Figure 2, and the power unit consists of lots of power electronics. The engine, the MGs, and the drive shaft are coupled with the mechanical parts, while the battery pack, the MGs, and the electric appliances are coupled with the power unit.

The principle of the EMT system can be illustrated as follows: one part of the engine's energy is transformed to electricity by the generator and then transmitted in the form of electrical power; the other part is directly transmitted by the planetary in the form of mechanical power. Afterwards, 


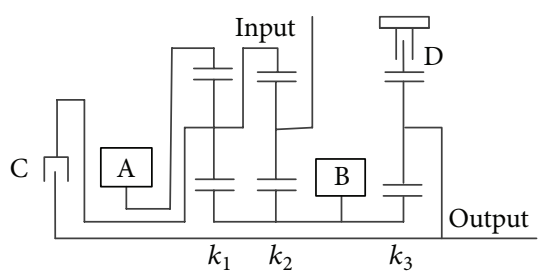

FIGURE 2: Main components of the dual-mode EMT system.

TABLE 1: Basic vehicle parameters.

\begin{tabular}{ll}
\hline Diesel engine & $\begin{array}{l}\text { Maximum power: } 295 \mathrm{~kW} / 223 \mathrm{rad} / \mathrm{s} \\
\text { Maximum torque: } 1427 \mathrm{Nm} / 168 \mathrm{rad} / \mathrm{s}\end{array}$ \\
\hline AC MGs & $\begin{array}{l}\text { Maximum power: } 78 \mathrm{~kW} \\
\text { Maximum torque: } 624 \mathrm{Nm}\end{array}$ \\
\hline Lithium battery & $\begin{array}{l}\text { Capacity: } 15 \mathrm{Ah} \\
\text { Bus voltage: } 550 \mathrm{~V}\end{array}$ \\
\hline Coupling mechanism & $k_{1}=2.13 ; k_{2}=2.13 ; k_{3}=2.13$ \\
\hline \multirow{2}{*}{ Gears } & Front-gear ratio: 1.43 \\
\hline \multirow{2}{*}{ Vehicle } & Rear-gear ratio: 4.15 \\
& Curb weight: $8105 \mathrm{~kg}$ \\
\hline
\end{tabular}

the motor transforms the electrical power from the generator or the battery pack into mechanical power, which would be the output to the drive shaft after converging with the mechanical power from the planetary. The battery pack is mainly used to compensate for the two MGs and the electric appliances' power deviation in the dynamic process and to meet the demand of electricity in some special conditions (e.g., when the engine starts).

Thus, the engine's power is transmitted through two paths in the EMT system: electrical path and mechanical path. As the engine's working points can be adjusted to the optimal region by the MGs, the fuel economy can be noticeably improved and the engine's power can be fully used to improve the driving performance of the vehicle. Additionally, the electricity demands from the appliances can be satisfied by the MGs, which transform the engine's power to electricity.

The research object of this paper is a heavy-duty vehicle, which is equipped with a dual-mode EMT system. The main components of the EMT system are three planetaries and two clutches $\mathrm{C}$ and $\mathrm{D}$, as shown in Figure 2.

The basic parameters of the HEV are shown in Table 1. The engine is equipped with a cooling fan, which is rated at $35 \mathrm{~kW}$. To save on the design cost, the parameters of the two MGs are completely the same. The cycle life of the battery pack has a close relationship with its charge and discharge rates, which are strictly restricted in this paper.

2.2. Nonlinear Characteristics. The control-oriented methods are utilized to analyze the nonlinear characteristics of the key components in the HEV. The fuel consumption rate map is shown in Figure 3, which is obtained from the experiment data of the engine.

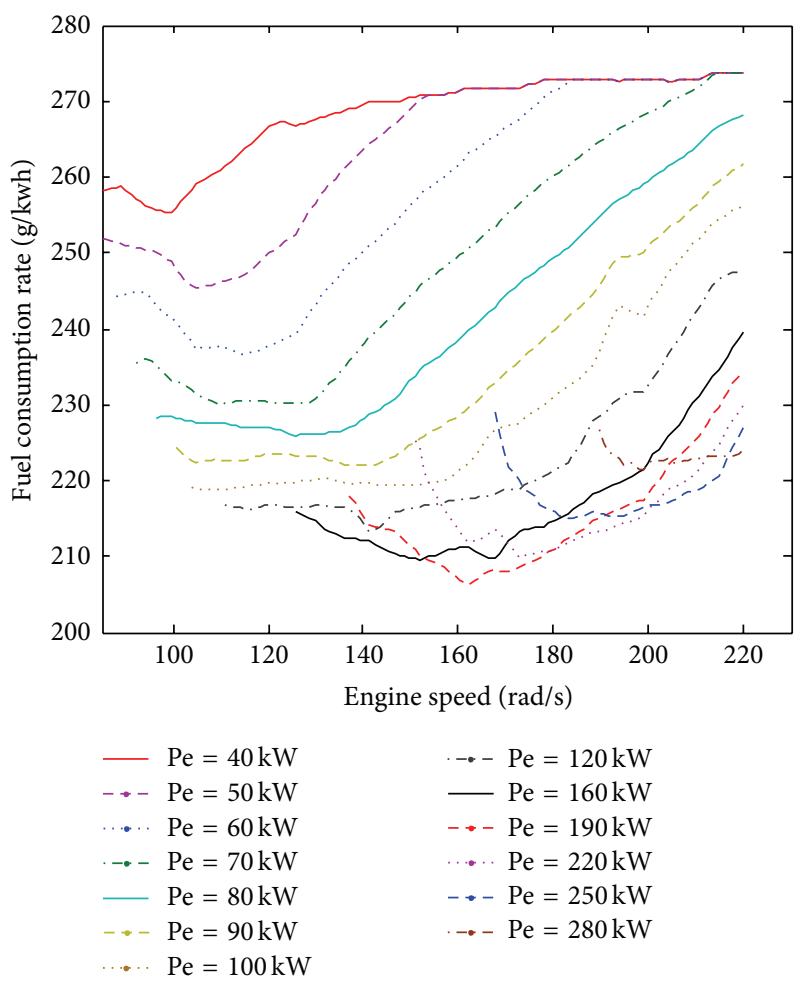

FIGURE 3: Fuel consumption rate under different powers of the engine.

It can be seen clearly that the engine's fuel consumption rate is a nonlinear function of the engine's speed under each power curve. To get the analytical expression of the fuel consumption rate, the polynomial fit method is adopted. The normalization processing to the engine's speed is carried out, with the following equations as

$$
\begin{array}{r}
\beta_{E}=\frac{\omega_{E}}{\omega_{E \max }}, \\
\text { fuel }\left(\beta_{E}\right)=\sum_{i=0}^{n} a_{i} \beta_{E}^{i},
\end{array}
$$

where $\omega_{E}$ and $\omega_{E \max }$ are the engine's actual speed and maximum speed, respectively, $\beta_{E}$ is the normalization variable of the engine's speed, fuel $\left(\beta_{E}\right)$ is the fuel consumption rate under the variable $\beta_{E}$, and $a_{i}$ is the fitting coefficient, $i=$ $1, \ldots, n$.

Moreover, the engine's maximum torque is also a nonlinear function of its speed. The data fitting is done by using the piecewise function as

$$
T_{E \max }\left(\omega_{E}\right)= \begin{cases}b_{0}+b_{1} \omega_{E} & 0 \leq \omega_{E} \leq \omega_{E 0} \\ c_{0}+c_{1} \omega_{E}+c_{2} \omega_{E}^{2} & \omega_{E 0} \leq \omega_{E} \leq \omega_{E \max }\end{cases}
$$

where $T_{E \max }\left(\omega_{E}\right)$ is the engine's maximum torque when its speed is $\omega_{E}, \omega_{E 0}$ is the engine's idling speed, and $b_{0}, b_{1}, c_{0}$, $c_{1}$, and $c_{2}$ are the fitting coefficients of the engine's external characteristic curve. 


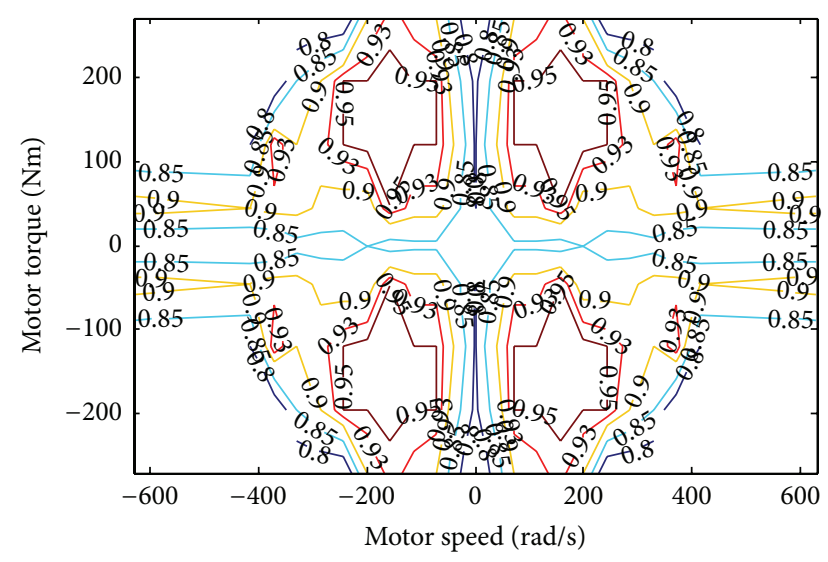

FIGURE 4: Efficiency map of the MGs.

The MGs' efficiency map, based on the experimental results, is shown in Figure 4. It is a nonlinear function of the MGs' torque and speed. As the four quadrants basically have the symmetric characteristic, taking the first quadrant as example, the surface fitting is carried out by using the method of least square with the expression

$$
\begin{aligned}
\lambda_{M} & =\frac{\omega_{M}}{\omega_{M \max }}, \\
\gamma_{M} & =\frac{T_{M}}{T_{M \max }}, \\
\eta_{M}\left(\lambda_{M}, \gamma_{M}\right) & =\sum_{i, j=0}^{n} b_{i j} \lambda_{M}^{i} \gamma_{M}^{j},
\end{aligned}
$$

where $\omega_{M}$ is the MGs' speed, including MGA and MGB, with $n_{M \max }$ as its maximum value, $T_{M}$ is the MGs' torque with $T_{M \max }$ as its maximum value, $\lambda_{M}$ and $\gamma_{M}$ are the normalization variables of the speed and the torque, with $\eta_{M}$ as their corresponding efficiency, and $b_{i j}$ is the fitting coefficient, $i, j=1, \ldots, n$.

The MGs have the characteristics of constant torque at low speed and constant power at high speed. The maximum torque is also a nonlinear function of the MGs' speed, as shown in the expression

$$
T_{M \max }\left(\omega_{M}\right)= \begin{cases}T_{M 0} & \left|\omega_{M}\right| \leq \omega_{M 0} \\ \frac{P_{M 0}}{\omega_{M}} & \omega_{M 0} \leq\left|\omega_{M}\right| \leq \omega_{M \max },\end{cases}
$$

where $T_{M \max }\left(\omega_{M}\right)$ is the maximum torque when the MGs' speed is $\omega_{M}$, and $\omega_{M 0}$ and $P_{M 0}$ are the rated speed and the rated power of the MGs, respectively.

The inherent resistance model is adopted to describe the working characteristics of the battery pack. The equation of its state of charge (SOC) can be written as

$$
\begin{aligned}
\frac{d}{d t} S(t) & =\frac{\sqrt{E_{b}^{2}-4 P_{b}(t) R_{b}}-E_{b}}{7200 C_{b} R_{b}}, \\
S(0) & =S_{0},
\end{aligned}
$$

where $E_{b}$ is the open-circuit voltage, $R_{b}$ is the inherent resistance, $C_{b}$ is the capacity (Ah), $P_{b}(t)$ is the power, $S(t)$ is the SOC, and $S_{0}$ is the initial value of the SOC.

The voltage of the DC bus is a vital parameter of the electrical system; the equation of which is presented as

$$
U(t)=\frac{\sqrt{E_{b}^{2}-4 P_{b}(t) R_{b}}+E_{b}}{2} .
$$

Thus, the SOC of the battery pack and the voltage of the DC bus are both nonlinear functions of the battery's power $P_{b}(t)$. Also, the inherent resistance $R_{b}$ and the opencircuit voltage $E_{b}$ are nonlinear functions of the SOC and the temperature. The nonlinear fitting could also be carried out by using the experimental data.

2.3. Coupling Interaction. If neglecting the elastic deformation and the gears' clearance of the coupling mechanism, the speed equation of the components can be presented in the form

$$
\left[\begin{array}{l}
\omega_{A} \\
\omega_{B}
\end{array}\right]=\left[\begin{array}{ll}
c_{11} & c_{12} \\
c_{21} & c_{22}
\end{array}\right]\left[\begin{array}{l}
\omega_{I} \\
\omega_{O}
\end{array}\right]
$$

where $\omega_{A}, \omega_{B}, \omega_{I}$, and $\omega_{O}$ represent the speed of MGA, MGB, the input shaft, and the output shaft, respectively, and $c_{11}, c_{12}$, $c_{21}$, and $c_{22}$ are the speed coefficients as determined by the operating mode of the EMT system and the parameters of the planetary.

When the clutch $\mathrm{C}$ is released and the brake $\mathrm{D}$ is engaged, the system operates in the EVT1 mode, with the speed coefficients as

$$
\begin{aligned}
& c_{11}=\frac{\left(1+k_{1}\right)\left(1+k_{2}\right)}{k_{1} k_{2}}, \\
& c_{12}=-\frac{\left(1+k_{1}+k_{2}\right)\left(1+k_{3}\right)}{k_{1} k_{2}}, \\
& c_{21}=0, \\
& c_{22}=1+k_{3},
\end{aligned}
$$

where $k_{1}, k_{2}$, and $k_{3}$ are the planetary gears' characteristic parameters that are determined by the ratio of gear teeth, as shown in Table 1 . When the clutch $C$ is engaged and the brake $\mathrm{D}$ is released, the system operates in the EVT2 mode, with the speed coefficients as

$$
\begin{aligned}
& c_{11}=-\frac{1+k_{2}}{k_{1}}, \\
& c_{12}=\frac{1+k_{1}+k_{2}}{k_{1}},
\end{aligned}
$$




$$
\begin{aligned}
& c_{21}=1+k_{2}, \\
& c_{22}=-k_{2} .
\end{aligned}
$$

The engine connects with the input shaft through the front gears, and the wheels connect with the output shaft through the rear gearbox. Their speed relations are

$$
\begin{aligned}
& \omega_{I}=\frac{\omega_{E}}{i_{f}}, \\
& \omega_{O}=\frac{v i_{r}}{r_{w}},
\end{aligned}
$$

where $i_{f}$ is the front-gear ratio, that is, the ratio from the engine to the input shaft, $v$ is the vehicle's speed, $r_{w}$ is the wheels' radius, and $i_{r}$ is the rear-gear ratio, that is, the ratio from the output shaft to the wheels.

As the rotary inertia of the planetary gears is relatively small and the power loss of the coupling mechanism is very little, the effects of these two factors are neglected, with the torque equation of the system as

$$
\left[\begin{array}{c}
T_{I} \\
T_{O}
\end{array}\right]=\left[\begin{array}{cc}
-c_{11} & -c_{21} \\
c_{12} & c_{22}
\end{array}\right]\left[\begin{array}{c}
T_{A} \\
T_{B}
\end{array}\right],
$$

where $T_{A}, T_{B}, T_{I}$, and $T_{O}$ represent the torques of MGA, MGB, the input shaft, and the output shaft, respectively.

The dynamic equations of the engine and the vehicle could be expressed as

$$
\begin{aligned}
J_{E} \frac{d \omega_{E}}{d t} & =T_{E}-\frac{T_{I}}{i_{f} \eta_{f}}, \\
m \frac{d v}{d t} & =\frac{T_{O} i_{r} \eta_{r}+T_{K}}{r_{w}}-F_{g},
\end{aligned}
$$

where $J_{E}$ is the rotary inertia of the engine, $T_{E}$ is the torque of the engine, $\eta_{f}$ is the transmission efficiency of the front gears, $m$ is the curb weight of the vehicle, $\eta_{r}$ is the transmission efficiency of the rear gearbox, $T_{K}$ is the braking torque on the wheels, and $F_{g}$ is the driving resistance from the ground and the air.

The power equation of the electrical components is

$$
P_{b}=T_{A} \omega_{A} \mu_{A}+T_{B} \omega_{B} \mu_{B}+P_{c},
$$

where $P_{c}$ is the total power of the electric appliances and $\mu_{A}$ and $\mu_{B}$ are the power factors of the MGs presented as

$$
\begin{aligned}
& \mu_{A}= \begin{cases}\frac{1}{\eta_{A}} & T_{A} \omega_{A} \geq 0 \\
\eta_{A} & T_{A} \omega_{A}<0,\end{cases} \\
& \mu_{B}= \begin{cases}\frac{1}{\eta_{B}} & T_{B} \omega_{B} \geq 0 \\
\eta_{B} & T_{B} \omega_{B}<0,\end{cases}
\end{aligned}
$$

where $\eta_{A}$ and $\eta_{B}$ are the efficiencies of the two MGs.

To sum up, the engine, the MGs, and the battery pack all have strong nonlinear characteristics, and they are coupled together by the EMT system. Therefore, the EMT system is a nonlinear-coupling system.

\section{Multiobjective Dynamic Optimization Model}

The standard form of a nonlinear dynamic optimization model can be presented as

$$
\begin{array}{ll}
\min & f(x, u, w, t), \\
\text { s.t. } & \dot{x}=g(x, u, w, t), \\
& h(x, u, w, t)=0, \\
& p(x, u, w, t) \leq 0,
\end{array}
$$

where $x, u, w$, and $t$ represent the state vector, the control vector, the random vector, and the time, $f(x, u, w, t)$ is cost function, $\dot{x}=g(x, u, w, t)$ is dynamic model of the system, and $h(x, u, w, t)=0$ and $p(x, u, w, t) \leq 0$ are equality and inequality constraints, respectively.

In the EMT system, the variables can be expressed as

$$
\begin{aligned}
& x=\left(\omega_{E}, \omega_{A}, \omega_{B}, v, S\right)^{T}, \\
& u=\left(T_{E}, T_{A}, T_{B}, T_{K}\right)^{T}, \\
& w=\left(F_{g}, P_{c}\right)^{T} .
\end{aligned}
$$

Thus, the state variables include the engine speed, the two MG speeds, the vehicle speed, and the battery SOC; the control variables include the engine torque, the two MG torques, and the braking torque; the random variables include the driving resistance and the power of the electric appliances, which can be expressed as

$$
\begin{gathered}
F_{g}=\widehat{F}_{g}+\delta F_{g}, \\
P_{c}=\widehat{P}_{c}+\delta P_{c},
\end{gathered}
$$

where $\widehat{F}_{g}$ and $\widehat{P}_{c}$ are the mean value of $F_{g}$ and $P_{c}, \delta F_{g}$, and $\delta P_{c}$ are white noises, with their mean value as

$$
\begin{gathered}
E\left(\delta F_{g}\right)=0, \\
E\left(\delta P_{c}\right)=0 .
\end{gathered}
$$

3.1. Multiple Optimization Objectives. The control strategy of the $\mathrm{HEV}$ is a multiobjective optimization problem with the cost functions as

$$
\begin{aligned}
& J_{D}=\int_{t_{0}}^{t_{0}+\Delta t} L_{D}[x(t), u(t), w(t), t] d t, \\
& J_{E}=\int_{t_{0}}^{t_{0}+\Delta t} L_{E}[x(t), u(t), w(t), t] d t, \\
& J_{F}=\int_{t_{0}}^{t_{0}+\Delta t} L_{F}[x(t), u(t), w(t), t] d t,
\end{aligned}
$$

where $L_{D}, L_{E}$, and $L_{F}$ are the index functions of the drivability, the current supply capacity, and the fuel economy, $t_{0}$ is the initial time, and $\Delta t$ is the time step. 
3.1.1. Drivability. The EMT system transmits the engine's power to the wheels to drive the vehicle. With the same power of the engine, which is determined by the driver's pedals, the more power is transmitted to the wheels; that is, the less the power will be lost, the better the vehicle's drivability will be. Therefore, the index function of the drivability can be expressed as

$$
L_{D}=P_{E}(t)-P_{D}(t)=T_{E}(t) \omega_{E}(t)-T_{O}(t) \omega_{O}(t) \eta_{r},
$$

where $P_{E}(t)$ is the power of the engine and $P_{D}(t)$ is the total power of the wheels at the time $t$.

The power $P_{D}(t)$ can be obtained from (10), (11), and (20), so the index function of $L_{D}$ is

$$
L_{D}=T_{E}(t) \omega_{E}(t)-\frac{i_{r} \eta_{r}}{3.6 r_{w}}\left[c_{12} T_{A}(t)+c_{22} T_{B}(t)\right] v(t) \text {. }
$$

Thus, the drivability is a nonlinear function of the state variables $\omega_{E}, v$ and control variables $T_{E}, T_{A}$, and $T_{B}$.

3.1.2. Current Supply Capacity. The EMT system can supply electrical power to meet the electricity demand of the appliances. The smaller the difference between the electricity supply and the demand is, the better the current supply capacity will be. Thus, the index function of the current supply capacity can be expressed as

$$
L_{E}=\left|P_{N}(t)-P_{A B}(t)\right|,
$$

where $P_{N}(t)$ is the electricity demand from the electric appliances and the battery pack and $P_{A B}(t)$ is the electricity supply from the two MGs.

To guarantee the cycle life of the battery pack, it is better to keep its SOC at around the ideal value. Thus, the power need of the battery pack is a function of its SOC, which can be represented by the cubic curve as

$$
P_{b 0}(t)=d_{b}\left[S_{\mathrm{idl}}-S(t)\right]^{3},
$$

where $P_{b 0}$ is the battery pack's power demand, $S_{\mathrm{idl}}$ is its ideal SOC, and $d_{b}$ is the charge-discharge factor.

The overall power demand from the battery pack and the electric appliances is presented in the form of

$$
P_{N}(t)=P_{b 0}(t)+P_{c}(t) .
$$

The two MGs can supply electricity at the same time, or one MG supplies electricity while the other is driving the vehicle. The overall power supply is expressed in the form of

$$
P_{A B}(t)=-T_{A}(t) \omega_{A}(t) \mu_{A}-T_{B}(t) \omega_{B}(t) \mu_{B},
$$

where $\mu_{A}$ and $\mu_{B}$ are the nonlinear functions of the MG's speed and torque, respectively. Besides, the power $T_{A}(t) \omega_{A}(t)$ and power $T_{B}(t) \omega_{B}(t)$ are defined to be positive when the MGs consume electricity; otherwise, they are negative when the MGs produce electricity.
Combining (22), (23), (24), and (25), the index function of the current supply capacity can be presented as

$$
\begin{aligned}
L_{E} & =\mid T_{A}(t) \omega_{A}(t) \mu_{A}+T_{B}(t) \omega_{B}(t) \mu_{B}+P_{c}(t) \\
& +d_{b}\left[S_{\mathrm{idl}}-S(t)\right]^{3} \mid .
\end{aligned}
$$

Thus, the current supply capacity is a nonlinear function of the state variables $\omega_{A}, \omega_{B}, S$, the control variables $T_{A}, T_{B}$, and the random variable $P_{c}$.

3.1.3. Fuel Economy. The less the engine's fuel consumption is, the better the fuel economy will be. Its index function is presented as

$$
L_{F}=T_{E}(t) \omega_{E}(t) \text { fuel }(t)
$$

Equation (1) shows the relationship between the fuel consumption and the engine's speeds under several power levels. The linear interpolation of the adjacent curves can be carried out as

$$
\begin{aligned}
& \text { fuel }(t) \\
& \begin{aligned}
= & \frac{T_{E} \omega_{E}-P_{E 2}}{P_{E 1}-P_{E 2}}\left[a_{10}+a_{11} \frac{\omega_{E}}{\omega_{E \max }}+a_{12}\left(\frac{\omega_{E}}{\omega_{E \max }}\right)^{2}\right] \\
& +\frac{T_{E} \omega_{E}-P_{E 1}}{P_{E 2}-P_{E 1}}\left[a_{20}+a_{21} \frac{\omega_{E}}{\omega_{E \max }}+a_{22}\left(\frac{\omega_{E}}{\omega_{E \max }}\right)^{2}\right],
\end{aligned}
\end{aligned}
$$

where $P_{E 1}$ and $P_{E 2}$ are the power values of the given curves beside $T_{E} \omega_{E}$.

Thus, the fuel economy is a nonlinear function of the state variable $\omega_{E}$ and the control variable $T_{E}$.

3.2. Dynamic Model of the EMT System. The usual form to present a dynamic model is

$$
\left(\begin{array}{c}
\frac{d x_{1}}{d t} \\
\frac{d x_{2}}{d t} \\
\vdots \\
\frac{d x_{n}}{d t}
\end{array}\right)=\left(\begin{array}{c}
f_{1}\left(t, x_{1}, x_{2}, \ldots, x_{n}, \bar{u}_{1}\right) \\
f_{2}\left(t, x_{1}, x_{2}, \ldots, x_{n}, \bar{u}_{2}\right) \\
\vdots \\
f_{n}\left(t, x_{1}, x_{2}, \ldots, x_{n}, \bar{u}_{n}\right)
\end{array}\right)
$$

where $\bar{x}=\left(x_{1}, x_{2}, \ldots, x_{n}\right)$ is the state vector, $t$ represents time, and $\bar{u}_{i}$ are vectors representing input functions.

In the EMT system, the variables have been identified after the dynamic optimization model (15), and the dynamic equations can be listed as

$$
\frac{d \omega_{E}(t)}{d t}=\frac{1}{J_{E}} T_{E}(t)+\frac{c_{11}}{J_{E} i_{f} \eta_{f}} T_{A}(t)+\frac{c_{21}}{J_{E} i_{f} \eta_{f}} T_{B}(t),
$$




$$
\begin{aligned}
& \frac{d \omega_{A}(t)}{d t}= \frac{c_{11}}{J_{E} i_{f}} T_{E}(t)+\left(\frac{c_{11}^{2}}{J_{E} i_{f}^{2} \eta_{f}}+\frac{c_{12}^{2} i_{r}^{2} \eta_{r}}{m r_{w}^{2}}\right) T_{A}(t) \\
&+\left(\frac{c_{11} c_{21}}{J_{E} i_{f}^{2} \eta_{f}}+\frac{c_{12} c_{22} i_{r}^{2} \eta_{r}}{m r_{w}^{2}}\right) T_{B}(t) \\
&+\frac{c_{12} i_{r}}{m r_{w}^{2}} T_{K}(t)-\frac{c_{12}^{2} i_{r}}{m r_{w}} F_{g}(t), \\
& \frac{d \omega_{B}(t)}{d t}= \frac{c_{21}}{J_{E} i_{f}} T_{E}(t)+\left(\frac{c_{11} c_{21}}{J_{E} i_{f}^{2} \eta_{f}}+\frac{c_{12} c_{22} i_{r}^{2} \eta_{r}}{m r_{w}^{2}}\right) T_{A}(t) \\
&+\left(\frac{c_{21}^{2}}{J_{E} i_{f}^{2} \eta_{f}}+\frac{c_{22}^{2} i_{r}^{2} \eta_{r}}{m r_{w}^{2}}\right) T_{B}(t) \\
&+\frac{c_{22} i_{r}}{m r_{w}^{2}} T_{K}(t)-\frac{c_{12} c_{22} i_{r}}{m r_{w}} F_{g}(t), \\
& \frac{d}{d t} S(t)= \frac{\sqrt{E_{b}^{2}-4 P_{b}(t) R_{b}-E_{b}}}{7200 C_{b} R_{b}}, \\
& \frac{F_{12} i_{r} \eta_{r}}{m r_{w}} T_{A}(t)+\frac{c_{22} i_{r} \eta_{r}}{m r_{w}} T_{B}(t)+\frac{1}{m r_{w}} T_{K}(t) \\
& d t \\
& \frac{1}{m}=
\end{aligned}
$$

where (30), (31), (32), and (33) are obtained by combining the kinematic equations (7) (12) and (34) is from the dynamic model (5) of the battery pack.

The initial conditions of the state variables are

$$
\begin{gathered}
\omega_{E}\left(t_{0}\right)=\omega_{E 0}, \\
\omega_{A}\left(t_{0}\right)=\omega_{A 0}, \\
\omega_{B}\left(t_{0}\right)=\omega_{B 0}, \\
v\left(t_{0}\right)=v_{0}, \\
S\left(t_{0}\right)=S_{0},
\end{gathered}
$$

where $\omega_{E 0}, \omega_{A 0}, \omega_{B 0}$, and $v_{0}$ can be collected by the sensors and $S_{0}$ can be obtained by the battery management system.

It can be seen from the dynamic equations that the dynamic models of the engine and the MGs are linear, while the dynamic model of the battery pack is nonlinear.

3.3. Equality and Inequality Constraints. The EMT system is a nonlinear-coupling system, and its constraints include both the single constraints of the components (inequality constraints) and the coupling constraints among the components (equality constraints).
3.3.1. Inequality Constraints. The engine, the MGs, and the battery must operate within their allowable ranges, as follows:

$$
\begin{aligned}
\omega_{E \min } & \leq \omega_{E}(t) \leq \omega_{E \max }, \\
T_{E \min }\left(\omega_{E}\right) & \leq T_{E}(t) \leq T_{E \max }\left(\omega_{E}\right), \\
\omega_{A \min } & \leq \omega_{A}(t) \leq \omega_{A \max }, \\
T_{A \min }\left(\omega_{A}\right) & \leq T_{A}(t) \leq T_{A \max }\left(\omega_{A}\right), \\
\omega_{B \min } & \leq \omega_{B}(t) \leq \omega_{B \max }, \\
T_{B \min }\left(\omega_{B}\right) & \leq T_{B}(t) \leq T_{B \max }\left(\omega_{B}\right), \\
S_{\min } & \leq S(t) \leq S_{\max }, \\
U_{\min } & \leq U(t) \leq U_{\max }, \\
T_{K}(t) & \leq T_{K \max }(v),
\end{aligned}
$$

where $\omega_{E \min }, \omega_{E \max }, \omega_{A \min }, \omega_{A \max }, \omega_{B \min }$, and $\omega_{B \max }$ are the minimum and maximum speeds of the engine, MGA and MGB, $T_{E \min }, T_{E \max }, T_{A \min }, T_{A \max }, T_{B \min }$, and $T_{B \max }$ are the minimum and maximum torques of the engine, MGA and MGB, which are nonlinear functions of their speeds, $S_{\min }$ and $S_{\max }$ are the minimum and maximum allowable values of the battery's SOC, $U_{\min }$ and $U_{\max }$ are the minimum and maximum allowable voltages of the $\mathrm{DC}$ bus, and $T_{K \max }$ is the maximum braking torque, which is a function of the vehicle speed.

Besides, as the torque response of the engine and the MGs requires a certain time, the control variables $T_{E}, T_{A}$, and $T_{B}$ must meet the extra constraints as follows:

$$
\begin{gathered}
T_{E 0} e^{-\left(t-t_{0}\right) / \tau_{E}}+T_{E \min }\left(1-e^{-\left(t-t_{0}\right) / \tau_{E}}\right) \leq T_{E}(t) \\
\leq T_{E 0} e^{-\left(t-t_{0}\right) / \tau_{E}}+T_{E \max }\left(1-e^{-\left(t-t_{0}\right) / \tau_{E}}\right), \\
T_{A 0} e^{-\left(t-t_{0}\right) / \tau_{A}}+T_{A \min }\left(1-e^{-\left(t-t_{0}\right) / \tau_{A}}\right) \leq T_{A}(t) \\
\leq T_{A 0} e^{-\left(t-t_{0}\right) / \tau_{A}}+T_{A \max }\left(1-e^{-\left(t-t_{0}\right) / \tau_{A}}\right), \\
T_{B 0} e^{-\left(t-t_{0}\right) / \tau_{B}}+T_{B \min }\left(1-e^{-\left(t-t_{0}\right) / \tau_{B}}\right) \leq T_{B}(t) \\
\leq T_{B 0} e^{-\left(t-t_{0}\right) / \tau_{B}}+T_{B \max }\left(1-e^{-\left(t-t_{0}\right) / \tau_{B}}\right),
\end{gathered}
$$

where $T_{E 0}, T_{A 0}$, and $T_{B 0}$ are the torques of the engine and the two MGs at the time $t_{0}$ and $\tau_{E}, \tau_{A}$, and $\tau_{B}$ are the time constraints of the corresponding components, which are assumed as first-order systems.

3.3.2. Equality Constraints. Combining (7) and (10), the speed relations among the MGs, the engine, and the vehicle can be obtained as

$$
\begin{aligned}
& \omega_{A}(t)=\frac{c_{11}}{i_{f}} \omega_{E}(t)+\frac{c_{12} i_{r}}{3.6 r_{w}} v(t), \\
& \omega_{B}(t)=\frac{c_{21}}{i_{f}} \omega_{E}(t)+\frac{c_{22} i_{r}}{3.6 r_{w}} v(t) .
\end{aligned}
$$




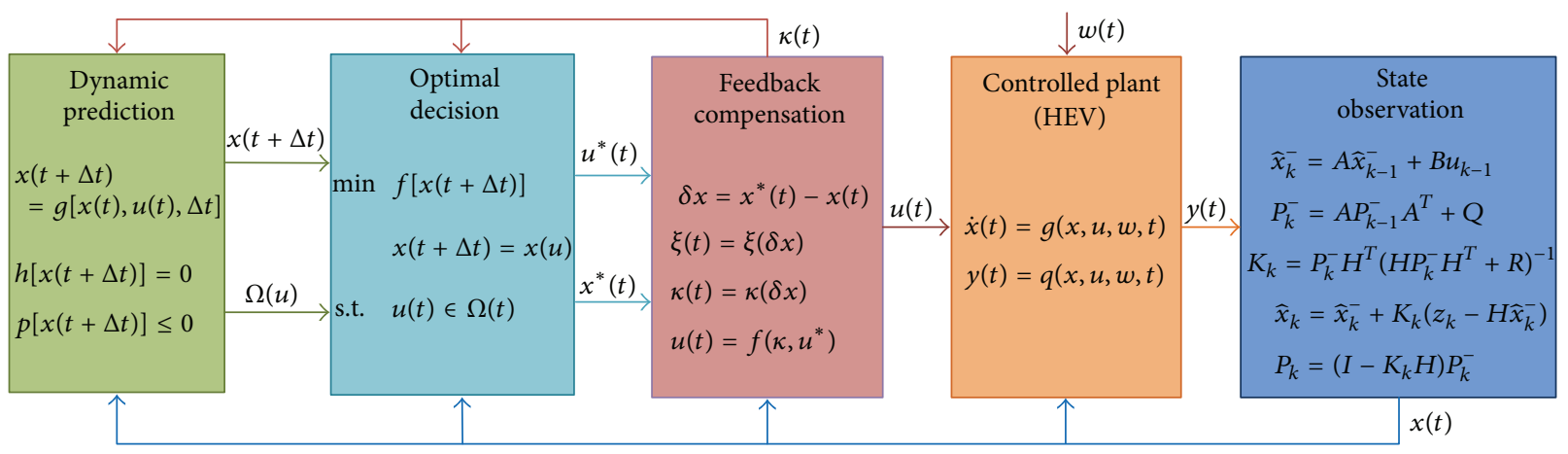

FIGURE 5: Control structure diagram of the OPDC approach.

In addition, (6) represents the relation between the bus voltage $U(t)$ and the battery pack's power $P_{b}(t)$ and (13) represents the relation between the two MGs, the battery pack, and the electric appliances' powers, which are also equality constraints.

\section{OPDC-Based Optimal Control Approach}

As the dynamic optimization model of the HEV has been derived in Section 2, the optimal control variables can be obtained by solving the model. However, the optimization model (15) has strong nonlinear-coupling and time-varying characteristics, so it is nearly impossible to solve the model in real time by using the existing methods. Therefore, a novel nonlinear control approach is proposed in this paper based on the characteristics of the system.

The dynamic optimization model of the HEV system has several characteristics: (1) the current states are available information; (2) the future states are functions of the control variables and the current states; (3) the cost functions, the inequality, and equality constraints can be expressed as functions of the future states; and (4) the future states will be influenced by the noise variables.

Due to the first characteristic, the current states can be obtained through the sensors or by using the state observation algorithm. The second characteristic indicates that the future states can be predicted by using the dynamic model of the system. Based on the third characteristic, the optimization model (15) can be simplified in the form of

$$
\begin{array}{ll}
\min & f[x(t+\Delta t)] \\
\text { s.t. } & x(t+\Delta t)=g[x(t), u(t), \Delta t], \\
& h[x(t+\Delta t)]=0 \\
& p[x(t+\Delta t)] \leq 0
\end{array}
$$

where $x(t)$ is the current state, which can be taken as a known information, and $x(t+\Delta t)$ is the future state after a time step $\Delta t$.

Moreover, because of the fourth characteristic, the impact of the noise needs to be compensated. Based on these characteristics of the dynamic optimization model of the $\mathrm{HEV}$ system, the novel nonlinear control approach proposed in this paper consists of the following four parts: state observation, dynamic prediction, optimal decision, and feedback compensation, as shown in Figure 5.

Firstly, the output variable $y(t)$ of the HEV system is collected by the sensors, so that the current state $x(t)$ can be estimated through the state observation algorithm. Secondly, the future state $x(t+\Delta t)$ is predicted, and the feasible region $\Omega(u)$ of the control variable $u(t)$ is obtained through the dynamic prediction algorithm based on the dynamic models and the constraints. Then, the optimal control variable $u^{*}(t)$ and its corresponding state variable $x^{*}(t+\Delta t)$ are achieved through the optimal decision algorithm based on the multiobjective optimization. Lastly, the feedback compensation to the model parameters and the control parameters is done by utilizing the deviation between the optimal state variable $x^{*}(t)$ and the actual state variable $x(t)$, and the final control variable $u(t)$ is applied to the HEV system.

4.1. State Observation. In the vehicle control area, such signals as the location, oil pressure, speed, and voltage can be collected by the sensors. As the torque sensor is too large, it is rarely used in the vehicles. Additionally, the driving resistance and SOC cannot be measured. Therefore, the state observation algorithm is utilized to obtain these variables.

As the response time of the two MGs (about $2 \mathrm{~ms}$ ) is shorter than the sampling time of the control system (20 ms), it can be assumed that the initial torques of the MGs approximate to their expected values

$$
\begin{aligned}
& T_{A 0} \approx T_{A}(k-1), \\
& T_{B 0} \approx T_{B}(k-1),
\end{aligned}
$$

where $T_{A}(k-1)$ and $T_{B}(k-1)$ are the control variables at the last sampling time.

Thus, the initial torques of the MGs are known information, which can be used to estimate the torque of the engine and the driving resistance of the vehicle.

The experimental results of the engine are shown in Figures 6 and 7. The diesel engine's expected speed is the control objective, while the actual speed follows. The engine's torque changes to realize the speed adjustment. The response time of the engine's torque is about $1200 \mathrm{~ms}$, which is much longer than the sampling time of the control system $(20 \mathrm{~ms})$. 


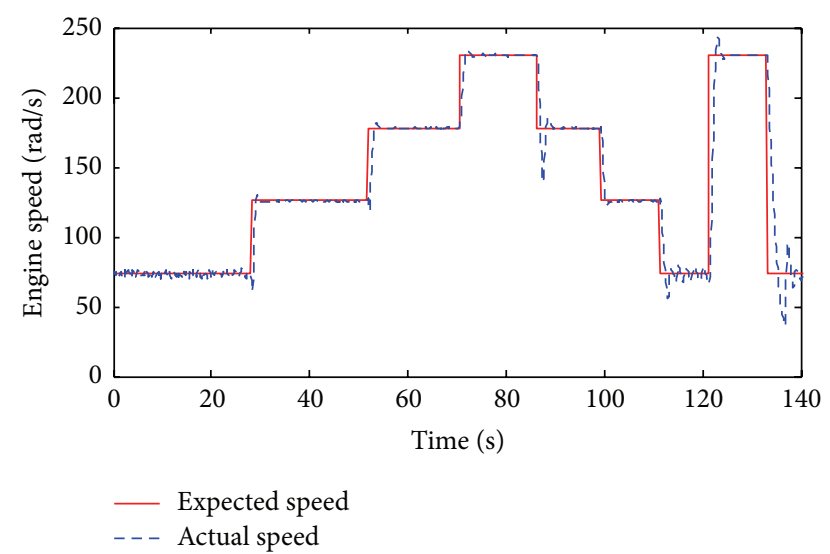

FIGURE 6: Speed results of the engine experiment.

Thus, the engine's torque changes a little during the sampling time. As a result, the state observation can be used to approximate the engine's actual torque, which is necessary to determine the range of the control variable $T_{E}$.

The Kalman filter is a set of mathematical equations that provide an efficient computational (recursive) means to estimate the state of a process, in a way that minimizes the mean of the squared error [31]. The state observation of the engine's torque and the vehicle's resistance was carried out in this paper based on the Kalman filtering algorithm.

The dynamic equations (30) (33) can be expressed in the discrete form of

$$
x_{0}(k)=A_{0} x_{0}(k-1)+B_{0} u_{0}(k-1),
$$

where $x_{0}=\left(\omega_{E}, \omega_{A}, \omega_{B}, v\right)^{T}$ is the speed vector, $u_{0}=\left(T_{E}\right.$, $\left.T_{A}, T_{B}, T_{K}, F_{g}\right)^{T}$ is the torque/force vector, and $A_{0}$ and $B_{0}$ are the speed and torque coefficient matrix as follows:

$$
\begin{aligned}
A_{0}=I_{4}=\left[\begin{array}{llll}
1 & 0 & 0 & 0 \\
0 & 1 & 0 & 0 \\
0 & 0 & 1 & 0 \\
0 & 0 & 0 & 1
\end{array}\right], \\
B_{0}=\left[\begin{array}{ccccc}
\frac{1}{J_{E}} & \frac{c_{11}}{J_{E} i_{f} \eta_{f}} & \frac{c_{21}}{J_{E} i_{f} \eta_{f}} & 0 & 0 \\
\frac{c_{11}}{J_{E} i_{f}} & \frac{c_{11}^{2}}{J_{E} i_{f}^{2} \eta_{f}}+\frac{c_{12}^{2} i_{r}^{2} \eta_{r}}{m r_{w}^{2}} & \frac{c_{11} c_{21}}{J_{E} i_{f}^{2} \eta_{f}}+\frac{c_{12} c_{22} i_{r}^{2} \eta_{r}}{m r_{w}^{2}} & \frac{c_{12} i_{r}}{m r_{w}^{2}} & -\frac{c_{12}^{2} i_{r}}{m r_{w}} \\
\frac{c_{21}}{J_{E} i_{f}} & \frac{c_{11} c_{21}}{J_{E} i_{f}^{2} \eta_{f}}+\frac{c_{12} c_{22} i_{r}^{2} \eta_{r}}{m r_{w}^{2}} & \frac{c_{21}^{2}}{J_{E} i_{f}^{2} \eta_{f}}+\frac{c_{22}^{2} i_{r}^{2} \eta_{r}}{m r_{w}^{2}} & \frac{c_{22} i_{r}}{m r_{w}^{2}} & -\frac{c_{12} c_{22} i_{r}}{m r_{w}} \\
0 & \frac{c_{12} i_{r} \eta_{r}}{m r_{w}} & \frac{c_{22} i_{r} \eta_{r}}{m r_{w}} & \frac{1}{m r_{w}} & -\frac{1}{m}
\end{array}\right] \Delta t .
\end{aligned}
$$

Based on the discrete equation (41) of the EMT system, the state equation and the output equation of the observation system can be obtained in the form of

$$
\begin{aligned}
& x_{1}(k)=A_{1} x_{1}(k-1)+B_{1} u_{1}(k-1)+w_{1}(k-1), \\
& y_{1}(k)=H x_{1}(k)+v_{1}(k),
\end{aligned}
$$

where $x_{1}=\left(\widehat{\omega}_{E}, \widehat{v}_{K}, \widehat{T}_{E}, \widehat{F}_{g}\right)^{T}$ is the state vector of the observation system, $u_{1}=\left(T_{A}, T_{B}, T_{K}\right)^{T}$ is the input vector, $w_{1}=\left(\delta \omega_{E}, \delta v, \delta T_{E}, \delta F_{g}\right)^{T}$ is the process noise vector, $y_{1}=$ $\left(\omega_{E}, \omega_{A}, \omega_{B}, v_{K}\right)^{T}$ is the output vector, $v_{1}=\left(\delta^{\prime} \omega_{E}, \delta^{\prime} \omega_{A}, \delta^{\prime} \omega_{B}\right.$, $\left.\delta^{\prime} v\right)^{T}$ is the measurement noise vector, and $A_{1}, B_{1}$, and $H_{1}$ are the system matrix, the input matrix, and the output matrix, which can be obtained by using the elements of matrix $A_{0}$ and $B_{0}$ as follows:

$$
\begin{aligned}
A_{1} & =\left[\begin{array}{cccc}
A_{0}(1,1) & A_{0}(1,4) & B_{0}(1,1) & B_{0}(1,5) \\
A_{0}(4,1) & A_{0}(4,4) & B_{0}(4,1) & B_{0}(4,5) \\
1 & 0 & 0 & 0 \\
0 & 1 & 0 & 0
\end{array}\right], \\
B_{1} & =\left[\begin{array}{ccc}
B_{0}(1,2) & B_{0}(1,3) & B_{0}(1,4) \\
B_{0}(4,2) & B_{0}(4,3) & B_{0}(4,4) \\
0 & 0 & 0 \\
0 & 0 & 0
\end{array}\right],
\end{aligned}
$$

$$
H_{1}=\left[\begin{array}{cccc}
1 & 0 & 0 & 0 \\
\frac{c_{11}}{i_{f}} & \frac{c_{12} i_{r}}{r_{w}} & 0 & 0 \\
\frac{c_{21}}{i_{f}} & \frac{c_{22} i_{r}}{r_{w}} & 0 & 0 \\
0 & 1 & 0 & 0
\end{array}\right] \text {. }
$$




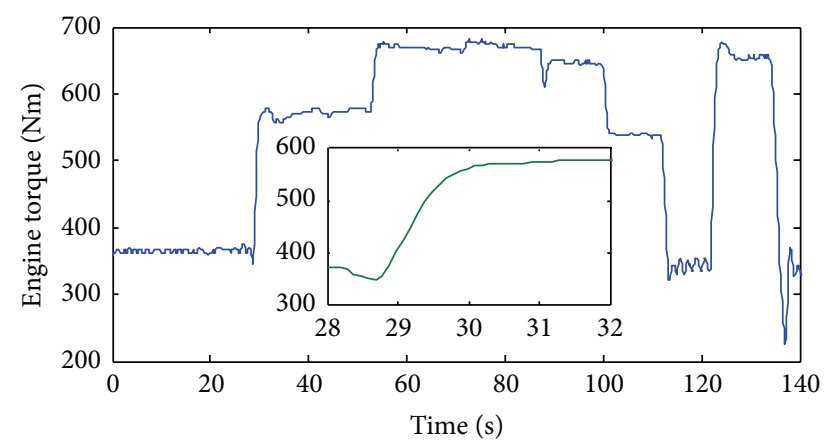

FIGURE 7: Torque results of the engine experiment.

Based on the observation system's state equation (43) and output equation (44), the engine's torque and the vehicle's resistance can be achieved by using the Kalman filtering algorithm. Moreover, the SOC of the battery pack can also be estimated using the Kalman filtering algorithm [32].

4.2. Dynamic Prediction. To obtain the future states and get the feasible region of the control variables, the dynamic prediction algorithm can be designed as follows.

Step 1. The torque ranges of the two MGs are calculated according to their current speeds, expressed as

$$
\begin{aligned}
& T_{A \max 0}\left(\widehat{\omega}_{A}\right)= \begin{cases}T_{A 0} & \left|\widehat{\omega}_{A}\right| \leq \omega_{A 0} \\
\frac{P_{A 0}}{\widehat{\omega}_{A}} & \omega_{A 0} \leq\left|\widehat{\omega}_{A}\right| \leq \omega_{A \max },\end{cases} \\
& T_{B \max 0}\left(\widehat{\omega}_{B}\right)= \begin{cases}T_{B 0} & \left|\widehat{\omega}_{B}\right| \leq \omega_{B 0} \\
\frac{P_{B 0}}{\widehat{\omega}_{B}} & \omega_{B 0} \leq\left|\widehat{\omega}_{B}\right| \leq \omega_{B \max },\end{cases} \\
& T_{A \min 0}\left(\widehat{\omega}_{A}\right)=-T_{A \max 0}\left(\widehat{\omega}_{A}\right), \\
& T_{B \min 0}\left(\widehat{\omega}_{B}\right)=-T_{B \max 0}\left(\widehat{\omega}_{B}\right),
\end{aligned}
$$

where $\widehat{\omega}_{A}$ and $\widehat{\omega}_{B}$ are the current speeds of the two MGs, which can be obtained by the sensors.

Step 2. The search ranges of the control variable $T_{A}$ are calculated according to its dynamic model, expressed as

$$
\begin{aligned}
& T_{A \max }=T_{A 0} e^{-\Delta t / \tau_{A}}+T_{A \max 0}\left(1-e^{-\Delta t / \tau_{A}}\right), \\
& T_{A \min }=T_{A 0} e^{-\Delta t / \tau_{A}}+T_{A \min 0}\left(1-e^{-\Delta t / \tau_{A}}\right) .
\end{aligned}
$$

Step 3. According to the control accuracy of the MGA, the linear search of the control variable $T_{A}$ is carried out, and its corresponding efficiency is calculated, expressed as

$$
\begin{aligned}
& T_{A p} \in\left[T_{A \min }, T_{A \max }\right], \quad p=1,2, \ldots, n_{1}, \\
& \eta_{A p}=\sum_{i, j=0}^{n} b_{i j}\left[\frac{\widehat{\omega}_{A}}{\omega_{A \max }}\right]^{i}\left[\frac{T_{A p}}{T_{A \max }}\right]^{j}, \\
& \mu_{A p}= \begin{cases}\frac{1}{\eta_{A p}} & T_{A p} \widehat{\omega}_{A} \geq 0 \\
\eta_{A p} & T_{A p} \widehat{\omega}_{A}<0,\end{cases}
\end{aligned}
$$

where $p$ is the serial number and $n_{1}$ is the amount of the search variables $T_{A p}$.

Step 4. According to the voltage constraints of the DC bus, the torque ranges of MGB are calculated, expressed as

$$
\begin{aligned}
P_{b \min } & =\frac{E_{b}}{R_{b}} U_{\max }-\frac{1}{R_{b}} U_{\max }^{2}, \\
P_{b \max } & =\frac{E_{b}}{R_{b}} U_{\min }-\frac{1}{R_{b}} U_{\min }^{2}, \\
T_{B \min 1} & = \begin{cases}\frac{P_{b \min }-\widehat{P}_{c}-T_{A p} \widehat{\omega}_{A} \mu_{A p}}{\widehat{\omega}_{B} \mu_{B}} & \widehat{\omega}_{B} \geq 0 \\
\frac{P_{b \max }-\widehat{P}_{c}-T_{A p} \widehat{\omega}_{A} \mu_{A p}}{\widehat{\omega}_{B} \mu_{B}} & \widehat{\omega}_{B}<0,\end{cases} \\
T_{B \max 1} & = \begin{cases}\frac{P_{b \max }-\widehat{P}_{c}-T_{A p} \widehat{\omega}_{A} \mu_{A p}}{\widehat{\omega}_{B} \mu_{B}} & \widehat{\omega}_{B} \geq 0 \\
\frac{P_{b \min }-\widehat{P}_{c}-T_{A p} \widehat{\omega}_{A} \mu_{A p}}{\widehat{\omega}_{B} \mu_{B}} & \widehat{\omega}_{B}<0,\end{cases}
\end{aligned}
$$

where $\widehat{P}_{c}$ is the current power of the electric appliances and $\mu_{B}$ is calculated by iterative algorithm.

Step 5. The research ranges of the control variable $T_{B}$ are calculated according to its dynamic model, expressed as

$$
\begin{aligned}
T_{B \max 2} & =T_{B 0} e^{-\Delta t / \tau_{B}}+T_{B \max 0}\left(1-e^{-\Delta t / \tau_{B}}\right), \\
T_{B \min 2} & =T_{B 0} e^{-\Delta t / \tau_{B}}+T_{B \min 0}\left(1-e^{-\Delta t / \tau_{B}}\right), \\
T_{B \min } & =\max \left[T_{B \min 1}, T_{B \min 2}\right], \\
T_{B \max } & =\min \left[T_{B \max 1}, T_{B \max 2}\right] .
\end{aligned}
$$

Step 6. According to the control accuracy of the MGB, the linear research of the control variable $T_{B}$ is carried out, and its corresponding efficiency is calculated, expressed as

$$
\begin{aligned}
& T_{B q} \in\left[T_{B \min }, T_{B \max }\right], \quad q=1,2, \ldots, n_{2}, \\
& \eta_{B q}=\sum_{i, j=0}^{n} b_{i j}\left[\frac{\widehat{\omega}_{B}}{\omega_{B \max }}\right]^{i}\left[\frac{T_{B q}}{T_{B \max }}\right]^{j}, \\
& \mu_{B q}= \begin{cases}\frac{1}{\eta_{B q}} & T_{B q} \widehat{\omega}_{B} \geq 0 \\
\eta_{B q} & T_{B q} \widehat{\omega}_{B}<0,\end{cases}
\end{aligned}
$$


where $q$ is the serial number and $n_{2}$ is the amount of the search variables $T_{B q}$.

Step 7. According to the dynamic model, the future speeds of the engine and the two MGs are predicted. The speed constraints are analyzed hierarchically, expressed as

$$
\begin{aligned}
\omega_{E}(t)= & \widehat{\omega}_{E}+\frac{1}{J_{E}} \widehat{T}_{E} \Delta t \\
& +\frac{1}{J_{E} i_{q} \eta_{q}}\left[c_{11} T_{A p}+c_{21} T_{B q}\right] \Delta t,
\end{aligned}
$$

constraint: $\omega_{E \min } \leq \widehat{\omega}_{E}(t) \leq \omega_{E \max }$;

$$
\begin{aligned}
v(t)= & \frac{i_{r} \eta_{r}}{m r_{w}}\left[c_{12} T_{A p}+c_{22} T_{B q}\right] \Delta t+\widehat{v} \\
& -\frac{1}{m} \widehat{F}_{g} \Delta t, \\
\omega_{A}(t)= & \frac{c_{11}}{i_{f}} \omega_{E}(t)+\frac{c_{12} i_{r}}{3.6 r_{w}} v(t),
\end{aligned}
$$

constraint: $\omega_{A \min } \leq \omega_{A}(t) \leq \omega_{A \max }$;

$$
\omega_{B}(t)=\frac{c_{21}}{i_{f}} \omega_{E}(t)+\frac{c_{22} i_{r}}{3.6 r_{w}} v(t)
$$

constraint: $\omega_{B \min } \leq \omega_{B}(t) \leq \omega_{B \max }$,

where $\widehat{v}$ is the current speed of the vehicle and $\widehat{T}_{E}$ and $\widehat{F}_{g}$ are the current engine torque and driving resistance, which have been obtained through the state observation.

Step 8. According to the dynamic model of the battery, the future SOC is calculated, and whether or not it meets the constraints is also judged, expressed as

$$
\begin{aligned}
P_{b}(t)= & \widehat{P}_{c}+T_{A p} \omega_{A}(t) \mu_{A p} \\
& +T_{B q} \omega_{B}(t) \mu_{B q}, \\
S(t)=\widehat{S} & +\frac{\sqrt{E_{b}^{2}-4 P_{b}(t) R_{b}}-E_{b}}{7200 C_{b} R_{b}} \Delta t,
\end{aligned}
$$

constraint: $S_{\min } \leq \widehat{S}(t) \leq S_{\max }$.

Step 9. If any of the constraints failed to be satisfied, the control variables are infeasible. Then the subsequent calculations are stopped, and the process turns to Step 10.

If all the constraints are fulfilled, the control variables are feasible. Then the control variables $T_{A p}$ and $T_{B q}$ are added to the feasible region $\Omega$, and the responding states are saved.
Step 10. Identify whether $q<n_{2}$.

If so, then $q=q+1$, and the process turns to Step 6 .

If not, identify whether $p<n_{1}$.

If so, then $p=p+1$, and turn to Step 3 .

If not, stop the calculation.

4.3. Optimal Decision. As the optimization objectives of the $\mathrm{HEV}$ system are all functions of the state variables and the sampling time of the controller is short enough, the integral functions (19) can be approximated using the trapezoid formula, expressed as

$$
\begin{aligned}
& J_{D}=\frac{L_{D}\left[x\left(t_{0}\right)\right]+L_{D}\left[x\left(t_{f}\right)\right]}{2}\left(t_{f}-t_{0}\right), \\
& J_{E}=\frac{L_{E}\left[x\left(t_{0}\right)\right]+L_{E}\left[x\left(t_{f}\right)\right]}{2}\left(t_{f}-t_{0}\right), \\
& J_{F}=\frac{L_{F}\left[x\left(t_{0}\right)\right]+L_{F}\left[x\left(t_{f}\right)\right]}{2}\left(t_{f}-t_{0}\right) .
\end{aligned}
$$

The current state $x\left(t_{0}\right)$ is a known information and the future state $x\left(t_{f}\right)=x\left(t_{0}+\Delta t\right)$ is a function of the control variable $u(t)$. Therefore, (54) can be rewritten in the form of

$$
\begin{aligned}
& J_{D}=L_{D}[x(t+\Delta t)]=f_{D}[x(u)], \\
& J_{E}=L_{E}[x(t+\Delta t)]=f_{E}[x(u)], \\
& J_{F}=L_{F}[x(t+\Delta t)]=f_{F}[x(u)] .
\end{aligned}
$$

As the feasible control variables and their corresponding future states have been obtained through dynamic prediction, the optimal decision turns to a steady multiobjective optimization problem with a feasible region of the optimization variables.

There are two ways to deal with the multiobjective optimization problem: the normalized and nonnormalized methods. The former transforms the multiple objectives to a single one through weight coefficients. The key problem is how to select the weight coefficients, which still depends on the engineer's experience. The latter directly handles the multiple optimization objectives by using the Pareto mechanism, through which a group of optimal solutions can be obtained. However, it is only suitable in solving the optimization problem of static parameters, rather than the optimal decision problem being discussed in this paper (which allows only one optimal solution).

According to the characteristics of the HEV system, a hierarchical optimization algorithm based on the normalized method is proposed [33], which turns the multiobjective 
optimization problem to several single-objective ones in different hierarchies:

\begin{tabular}{|c|c|c|c|c|}
\hline $\begin{array}{l}\min L_{F}[x(u)] \longrightarrow \omega_{E}^{*} \\
\text { if }\left|\omega_{E}(t)-\omega_{E}^{*}\right| \leq \delta \omega_{0} \\
\Omega_{2}(u)=\Omega(u) \\
\text { else if } \omega_{E}(t)<\omega_{E}^{*} \\
\Omega_{2}(u)=\Omega(u) \text { except } \\
\omega_{E}(t+\Delta t)<\omega_{E}(t) \\
\text { else } \\
\Omega_{2}(u)=\Omega(u) \text { except } \\
\omega_{E}(t+\Delta t)>\omega_{E}(t)\end{array}$ & $\stackrel{\Omega_{2}}{\longrightarrow}$ & $\begin{array}{l}\min \quad L_{E}[x(u)] \longrightarrow L_{E}^{\min } \\
\text { s.t. } \quad u \in \Omega_{2}(u) \\
\text { if } L_{E}^{\min }>\delta E \\
L_{E}^{\min } \longrightarrow x^{*}(u) \longrightarrow u^{*}(t) \\
\text { else } \\
\Omega_{3}(u)=\Omega_{2}(u) \text { except } \\
L_{E}\left[x\left(u_{i}\right)\right] \leq \delta E \\
\text { end }\end{array}$ & $\stackrel{\Omega_{3}}{\longrightarrow}$ & $\begin{array}{l}\min L_{D}[x(u)] \\
\text { if } \Omega_{3}(u) \neq \phi \\
\min L_{D}[x(u)] \\
\text { s.t. } u \in \Omega_{3}(u) \\
x^{*}(u) \longrightarrow u^{*}(t) \\
\text { else } \\
\text { end }\end{array}$ \\
\hline
\end{tabular}

(c) Drivability

(a) Fuel economy

(b) Current supply capacity

Hierarchical optimization algorithm

Firstly, the engine's optimal speed $\omega_{E}^{*}$ is achieved based on the fuel economy optimization, and it is compared with the current speed $\omega_{E}(t)$. Having the two speed levels close enough illustrates that the fuel economy is already good, so there is no need to adjust the engine's speed. If not, the feasible region should be restricted to ensure that the engine's future speed moves toward the optimal one.

Secondly, the current supply capacity is optimized in the new feasible region $\Omega_{2}(u)$, through which the minimum $L_{E}^{\min }$ is obtained. Having $L_{E}^{\mathrm{min}}$ larger than the threshold means that the current supply capacity is bad and needs to be optimized. If $L_{E}^{\mathrm{min}}$ is smaller than the threshold, it means that a good current supply capacity can be achieved under some of the control variables. Adding these control variables into the new feasible region $\Omega_{3}(u)$ and turning to the next optimization hierarchy then follow.

Lastly, the drivability is optimized in the new feasible region $\Omega_{3}(u)$. As $\Omega_{3}(u)$ has ensured the fuel economy and the current supply capacity, the optimal comprehensive performance can be achieved in this hierarchy.

4.3.1. Fuel Economy Hierarchy. The expected power of the engine is determined by the driver's pedal, which can be explained as

$$
P_{E \text { com }}=f_{\alpha}(\alpha)= \begin{cases}\alpha^{2} P_{E \max } & \alpha \geq 0 \\ P_{E \mathrm{idl}} & \alpha<0,\end{cases}
$$

where $\alpha$ is the position of the driver's pedal and $P_{E \text { com }}, P_{E \max }$, and $P_{\text {Eidl }}$ represent the engine's expected power, maximum power, and idle power, respectively.
As shown in Figure 3, the bottom point of each curve represents the engine's optimal speed under the corresponding power. Taking $P_{E 1}$, for example, while

$$
\frac{d \text { fuel }}{d \omega_{E}} \mid P_{E 1}=a_{11} \frac{1}{\omega_{E \max }}+2 a_{12} \frac{\omega_{E}}{\omega_{E \max }^{2}}=0,
$$

the optimal speed can be obtained as $\omega_{E 1}^{*}=$ $-\left(a_{11} / 2 a_{12}\right) \omega_{E \max }$.

Using the same method, the optimal speed levels under a group of engine's powers are found out in advance, and the optimal speed curve can then be plotted as shown in Figure 8.

Thus, the engine's optimal speed is a function of the expected power, which can be obtained through three-time fitting of the polynomial expression

$$
\begin{aligned}
& \omega_{E}^{*}=e_{0}+e_{1} \frac{P_{E \mathrm{com}}}{P_{E \max }}+e_{2}\left(\frac{P_{E \mathrm{com}}}{P_{E \max }}\right)^{2}+e_{3}\left(\frac{P_{E \mathrm{com}}}{P_{E \max }}\right)^{3}, \\
& \omega_{E}^{*}=\max \left(\omega_{E}^{*}, \omega_{E \min }\right), \\
& \omega_{E}^{*}=\min \left(\omega_{E}^{*}, \omega_{E \max }\right) .
\end{aligned}
$$

The fitting coefficients are

$$
\begin{aligned}
& e_{0}=58.4, \\
& e_{1}=413.5, \\
& e_{2}=-663.9, \\
& e_{4}=419.3 .
\end{aligned}
$$

4.3.2. Current Supply Capacity Hierarchy. As the sampling time is relatively short, the variables $\omega_{A}, \omega_{B}, \mu_{A}, \mu_{B}, S$, and $P_{c}$ are approximated to be unchanged during the time interval. 


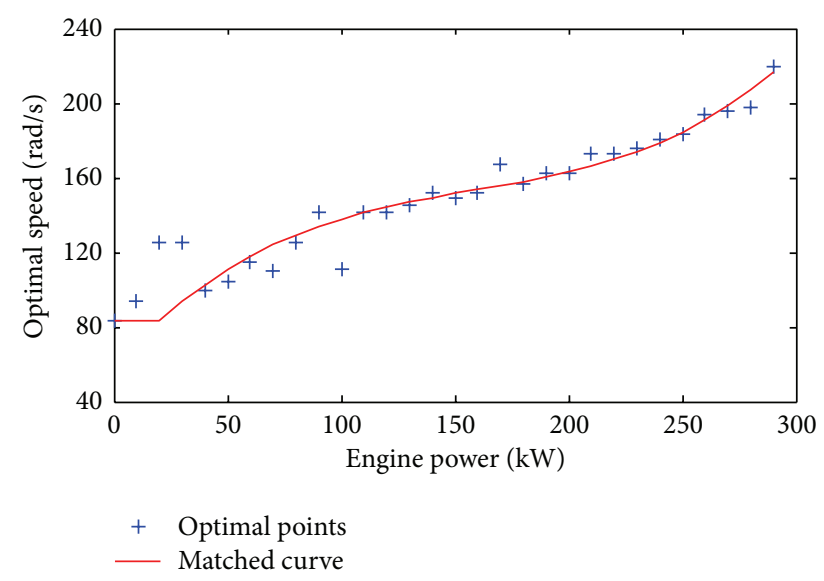

FIGURE 8: Optimal speed curve of the engine.

Then, the current supply capacity becomes the function of the control variables $T_{A}$ and $T_{B}$. By making $L_{E}=0$ in (26), the equality relation of $T_{A}$ and $T_{B}$ can be achieved, expressed as

$$
T_{B}=\frac{d_{b}\left(S-S_{\mathrm{idl}}\right)^{3}-P_{c}}{\omega_{B} \mu_{B}}-\frac{\omega_{A} \mu_{A}}{\omega_{B} \mu_{B}} T_{A} .
$$

In the feasible region $\Omega_{2}(u)$, as for each control variable $T_{A}$, the optimal torque $T_{B}^{*}$ can be calculated through (61). The points that fulfill $L_{E} \leq \delta E$ will be determined by searching beside the optimal torque $T_{B}^{*}$, which are then added up to the new feasible region $\Omega_{3}(u)$. If neither of the points meet the conditions mentioned above, the smallest point of $L_{E}$ will be taken as the optimal decision.

4.3.3. Drivability Hierarchy. As shown in (21), the drivability $L_{D}$ is a nonlinear function of the variables $\omega_{E}, v, T_{E}, T_{A}$, and $T_{B}$. Based on the feasible region of these variables, which has been achieved previously, the solutions to the nonlinear optimization function (21) can be obtained by using the feasible directions method, which is a gradient-based nonlinear programming algorithm [34].

The basic idea of the feasible directions method is as follows: start the search from a feasible point, and search in the feasible descent direction to find a new feasible point that makes the cost function fall down until the minimum value of the cost function is determined.

As the states $\omega_{E}, v$, and $T_{E}$ change slowly, their influence can be ignored in a sampling time. The partial derivative of $L_{D}$ with respect to $T_{A}$ and $T_{B}$ can be obtained as

$$
\begin{aligned}
& \frac{\partial L_{D}}{\partial T_{A}}=-c_{12} \frac{i_{r} \eta_{r}}{3.6 r_{w}} v(t), \\
& \frac{\partial L_{D}}{\partial T_{B}}=-c_{22} \frac{i_{r} \eta_{r}}{3.6 r_{w}} v(t) .
\end{aligned}
$$

The gradient of $L_{D}$ is $\nabla L_{D}=\left[\partial L_{D} / \partial T_{A}, \partial L_{D} / \partial T_{B}\right]^{T}$.

Based on the gradient $\nabla L_{D}$, the drivability can be optimized by utilizing the feasible directions method. The optimization process is as follows.
Step 1. Select an initial feasible point in the feasible region $\Omega_{3}(u)$, and search in the negative gradient direction to find a new feasible descent point.

In the EVT1 mode, $c_{12}<0, c_{12}<0$, so

$$
\begin{aligned}
& \frac{\partial L_{D}}{\partial T_{A}}>0, \\
& \frac{\partial L_{D}}{\partial T_{B}}<0,
\end{aligned}
$$

which means that $L_{D}$ is monotonously increasing with $T_{A}$ and monotonously decreasing with $T_{B}$. Therefore, the decreasing direction of $T_{A}$ and the increasing direction of $T_{B}$ are the negative gradient directions (descent directions).

In the EVT2 mode, $c_{12}>0, c_{22}<0$, so

$$
\begin{aligned}
& \frac{\partial L_{D}}{\partial T_{A}}<0, \\
& \frac{\partial L_{D}}{\partial T_{B}}>0
\end{aligned}
$$

which means that $L_{D}$ is monotonously decreasing with $T_{A}$ and monotonously increasing with $T_{B}$. Therefore, the increasing direction of $T_{A}$ and the decreasing direction of $T_{B}$ are the negative gradient directions (descent directions).

Step 2. Search along the negative gradient direction step by step to find all the optimization boundary points.

In the EVT1 mode, find the points that satisfy the condition that $T_{B}$ is the largest under the same $T_{A}$, while $T_{A}$ is the smallest under the same $T_{B}$.

In the EVT2 mode, find the points that satisfy the condition that $T_{B}$ is the smallest under the same $T_{A}$, while $T_{A}$ is the largest under the same $T_{B}$.

Step 3. Calculate the function $L_{D}$ of the optimization boundary points, find the minimum value $L_{D}^{\mathrm{min}}$, and its corresponding control variables are the optimal decision.

In addition, as the optimal decision algorithm is used to find out the optimal control variables in the feasible region, which has been obtained through the dynamic prediction, the iterative computation is unnecessary at each step time. Therefore, the convergence of the optimization algorithm can be ensured, which is important for the control system.

4.4. Feedback Compensation. As the OPDC approach is a model-based control method, its control effects are related to the accuracy of the model. Additionally, the external disturbances and noises could also make the components' actual states deviate from the expected states, which has a serious impact on the control effect. To overcome the influence of the model error and the disturbance signals, the model parameters and control parameters can be modified by utilizing the state feedback, which is also called as the feedback compensation.

The engine's speed is an important control variable and state variable. To guarantee its control accuracy, the closedloop control is carried out according to the deviation between 


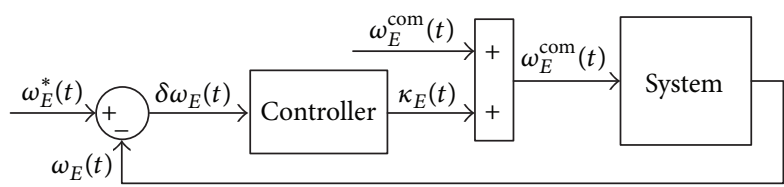

FIGURE 9: Feedback compensation of the engine's speed.

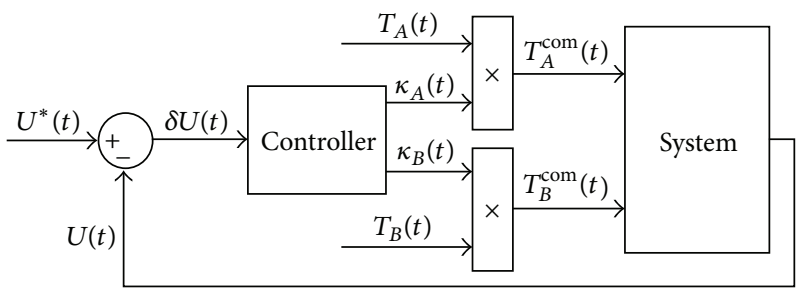

FIGURE 10: Feedback compensation of the DC bus voltage.

the optimal speed and the actual speed, which is illustrated in Figure 9.

The feedback compensation to the engine's expected speed $\omega_{E}^{\text {com }}(t)$ is based on the PID controller

$$
\begin{aligned}
& \delta \omega_{E}(t)=\omega_{E}^{*}(t)-\omega_{E}(t) \\
& \kappa_{E}(t) \\
& \quad=K_{P}\left[\delta \omega_{E}(t)+\frac{1}{T_{1}} \int_{0}^{t} \delta \omega_{E}(t) d t+T_{D} \frac{d}{d t} \delta \omega_{E}(t)\right], \\
& \omega_{E}^{\text {com }}(t)=\omega_{E}^{\text {com }}(t)+\kappa_{E}(t),
\end{aligned}
$$

where $\kappa_{E}(t)$ is the compensation value and $K_{P}, T_{1}$, and $T_{D}$ are the parameters of the PID controller.

The bus voltage is an important parameter of the electrical system; the control accuracy of which directly determines whether or not the system would operate normally. To realize the accurate control of the bus voltage, the feedback compensation to the torque commands of the two MGs is carried out by using the deviation between the expected voltage and the actual voltage (see Figure 10). As the compensation of the MGs' torque would make the control variables deviate from the optimal points and further influence the comprehensive performance of the system. Therefore, if the bus voltage is within the allowable range, it is better not to modify the control variables. Only when the voltage deviation is relatively large, which may influence the system's normal operation, it is necessary to make small modification to the control variables. The amount of compensation can be obtained through experimental test and calibration.

\section{Simulation and Real-Time Implementation}

The dynamic simulation model and the on-line test platform of the HEV equipped with an EMT system are created to verify the control effects and the real-time performance of the nonlinear optimal control approach being proposed in this paper.

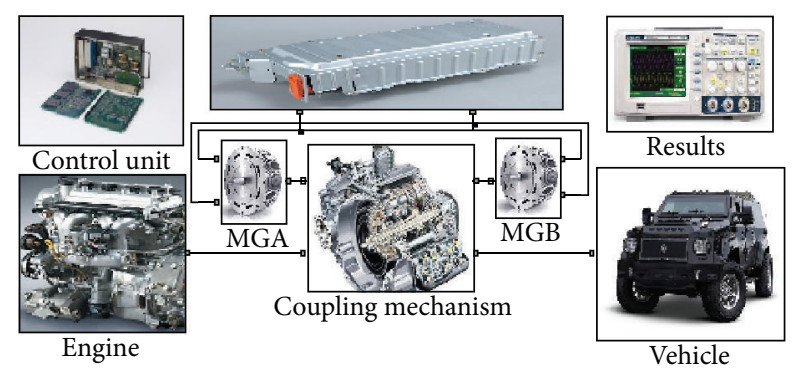

FIgURE 11: Dynamic simulation platform of the HEV.

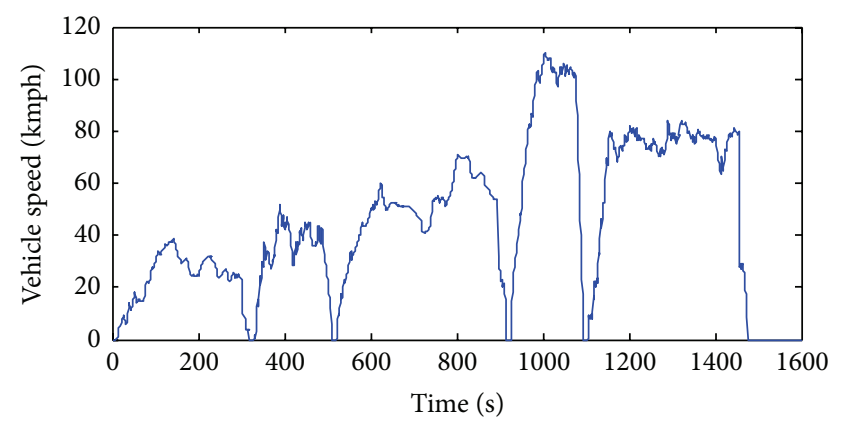

FIGURE 12: Driving cycle of the heavy-duty HEV.

5.1. Dynamic Simulation. The simulation model is built on the MATLAB platform (see Figure 11). The model mainly consists of the physical system and the control system. The actual working characteristics of the physical system are fully considered in the model. The experimental data of the engine, the MGs, the battery, and the coupling mechanism are used to truly reflect the operation condition of the physical system.

In the control system, the control algorithm is written by using the embedded MATLAB function. The inputs are the state variables collected by the sensors, and the outputs are the control variables calculated by the control algorithm. As the MATLAB function can be transformed to $C$ code and downloaded to the real controller, the development time of the control system can be reduced significantly.

The driving cycle of the heavy-duty HEV being studied in this paper is shown in Figure 12, which is derived from the experimental data. The resistance cycle and the electrical power cycle are also designed based on the characteristics of the studied vehicle, as shown in Figure 13. The vehicle's driving resistance, including the rolling resistance, the air resistance, and the gradient resistance, can be expressed with a unified resistance coefficient. The electricity demand of the electric appliances, including cooling fans, grease pump, air conditioning units, and so forth, can be expressed with a unified electrical power. To verify the control effects and the adaptability of the OPDC approach, both the resistance cycle and the electrical power cycle are changed with the vehicle speed, which both have the characteristic of randomness.

The rule-based control approach, being used for comparison, is also written and simulated using the same simulation platform and cycles. 


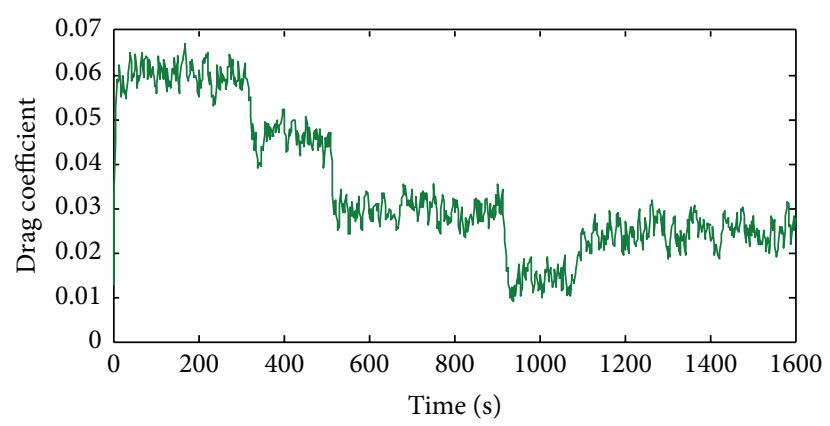

(a) Resistance cycle

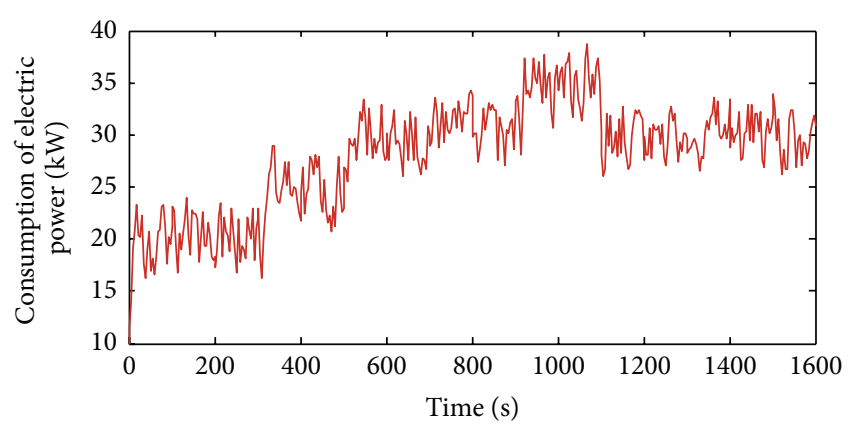

(b) Electrical power cycle

FIGURE 13: Resistance cycle and electrical power cycle of the HEV.

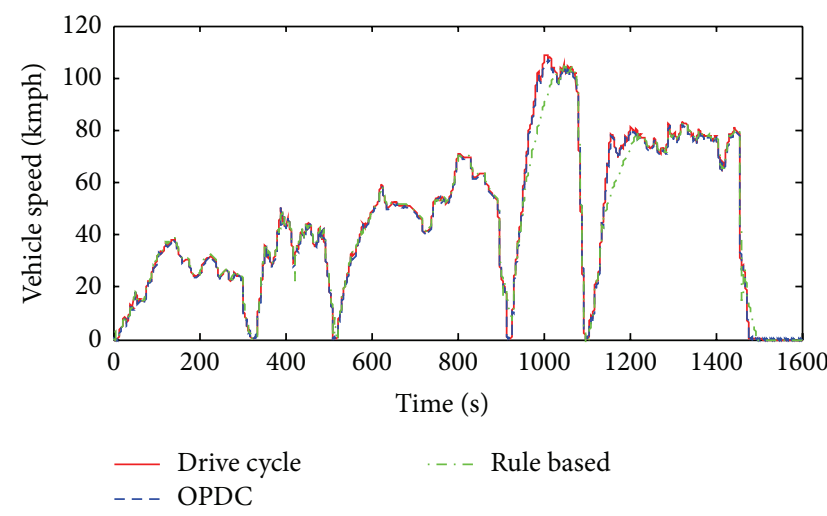

(a) Vehicle speed

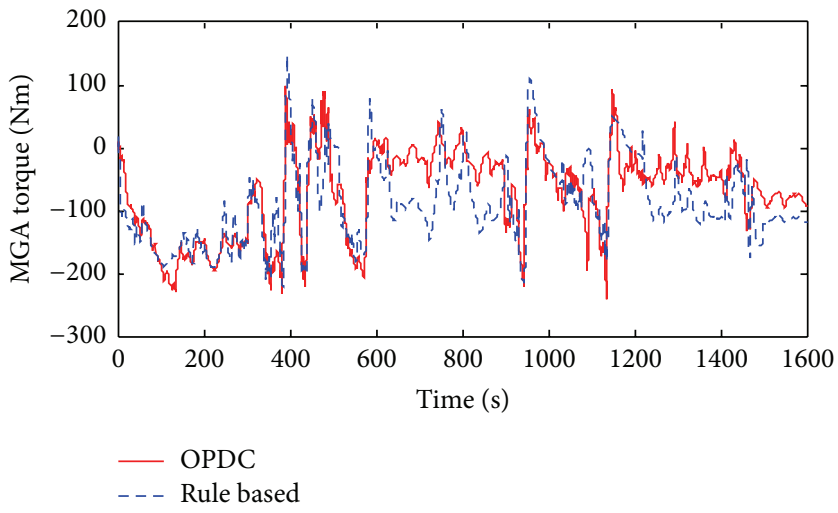

(c) MGA torque

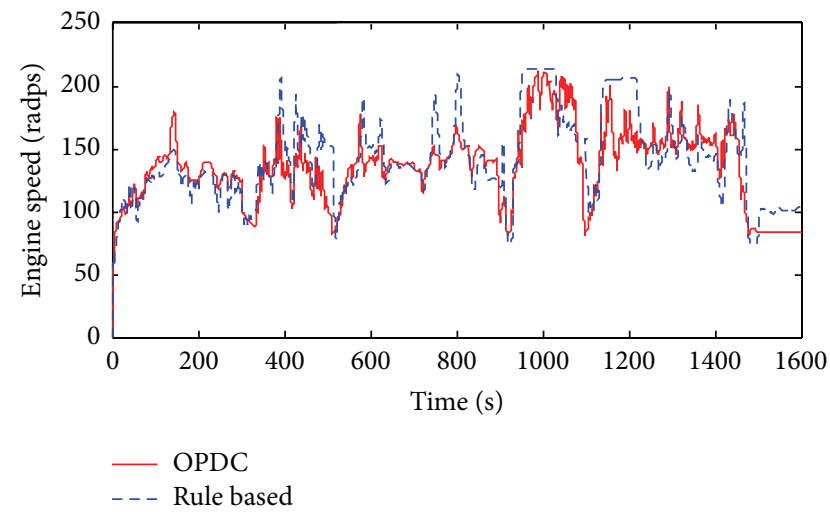

(b) Engine speed

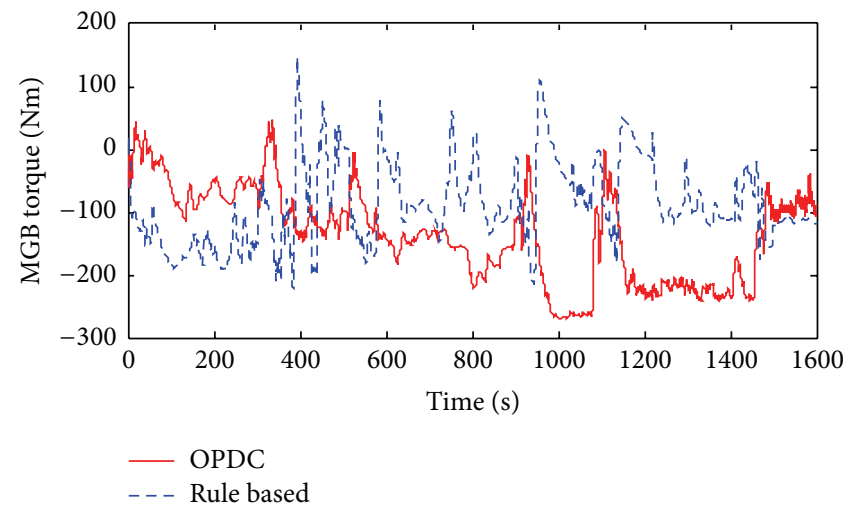

(d) MGB torque

FIGURE 14: Simulation results under the comprehensive cycles.

The simulation results under the comprehensive cycles are shown in Figure 14. The vehicle speed under the OPDC approach is in good agreement with the driving cycle, which illustrates that a good driving performance is achieved by this control approach. The vehicle's speed under the rule-based approach matches the driving cycle as good most of the time, but when the vehicle is accelerating fast, it cannot deliver a good driving performance. Thus, the OPDC approach can achieve better drivability than the rule-based approach. It can be seen from (b) that the engine's speeds under the two approaches have similar trends, but the values are not the same. This is because the EMT system has two degrees of freedom, which illustrates that the engine's speed can be optimized when the vehicle's speed is restrained. From (c) and (d), we know that the MGs' torques under the two approaches are quite different, especially the MGB's torques. This is because the torques are obtained by considering the comprehensive performance under the OPDC approach, but only the experience of the engineers under the rule-based approach. In summary, the speeds and torques under the two 


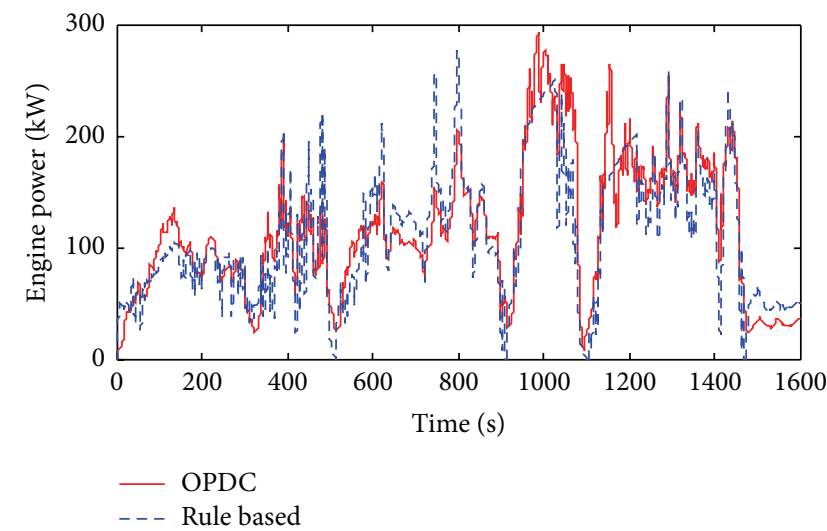

(a) Engine power

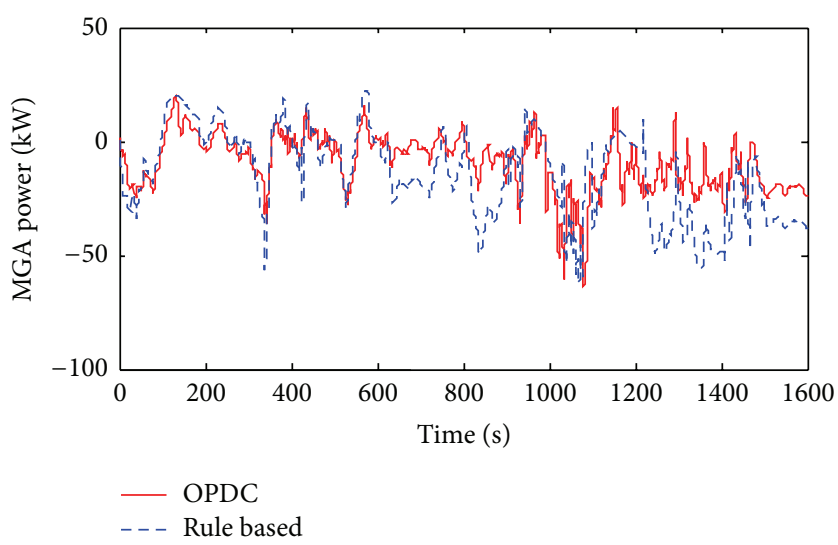

(c) MGA power

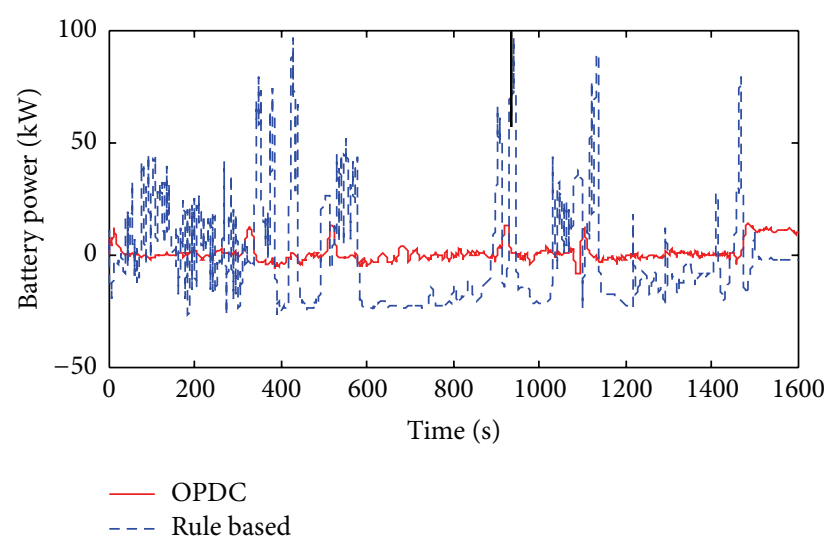

(b) Battery pack power

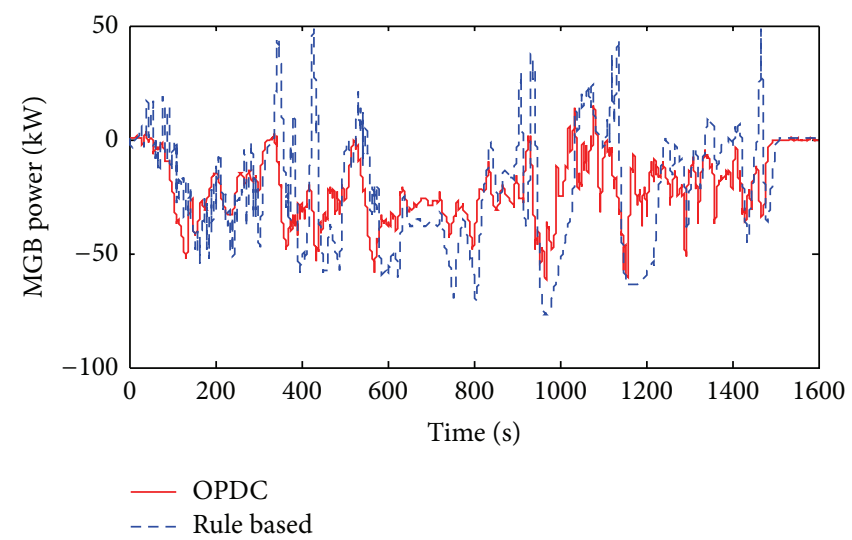

(d) MGB power

FIGURE 15: Comparison of the components' power.

control approaches are different, which leads to difference in performance.

Figure 15 shows the power states of the engine, the battery pack, and the two MGs under the two control approaches. It can be seen from (a) that the engine's powers are close most of the time, but when the road is bad or the vehicle's speed is high (see Figures 12 and 13), the engine's power under the OPDC approach is obviously higher than that under the rule-based approach. That is to say, the OPDC approach can achieve a better driving performance. It can be seen from (b) that the battery's power under the OPDC approach is much smaller and steadier than that under the rule-based approach. Therefore, the OPDC approach can avoid battery overcharging or overdischarging and greatly improve the battery's cycle life. From (c) and (d), we can see that the MGs' powers under the two approaches have similar trends, but the powers under the OPDC approach are a little smaller, which can reduce the conversion loss between the electrical and mechanical powers and improve the comprehensive efficiency of the EMT system.

The states of the electrical system are shown in Figure 16. It can be seen from (a) that the SOC basically remains unchanged under the OPDC approach, which reduces the loss during the charge-discharge process and reduces the pressure to the battery pack. The SOC can be as close as the initial state under the rule-based method after repeatedly modifying the control parameter, which provides the same condition for the comparison of the two control approaches. However, if the modification is not carried out according to the given cycles, the rule-based method can hardly guarantee the recovery of the SOC. Therefore, it gives more pressure to the battery pack, which needs to have more energy storage. It can be seen from (b) that the voltage of the DC bus under the OPDC approach is much steadier than that under the rule-based approach. This is because the OPDC approach has made a prediction to the bus voltage and ensured the balance between the supply and the demand of the electrical power, which cannot be done under the rule-based approach.

Figure 17 shows the comparison of the engine's working points under the two control approaches. It can be seen from the comparisons of (a) and (b) that the working points under the OPDC approach concentrate near the engine's optimal fuel economy curve, while the working points under the rule-based approach are mostly scattered near the optimal fuel economy region, which is also the factor needed to be considered in the process of rule-making. To guarantee the drivability and the current supply capacity, the working points of the engine deviate from the optimal economic curve 


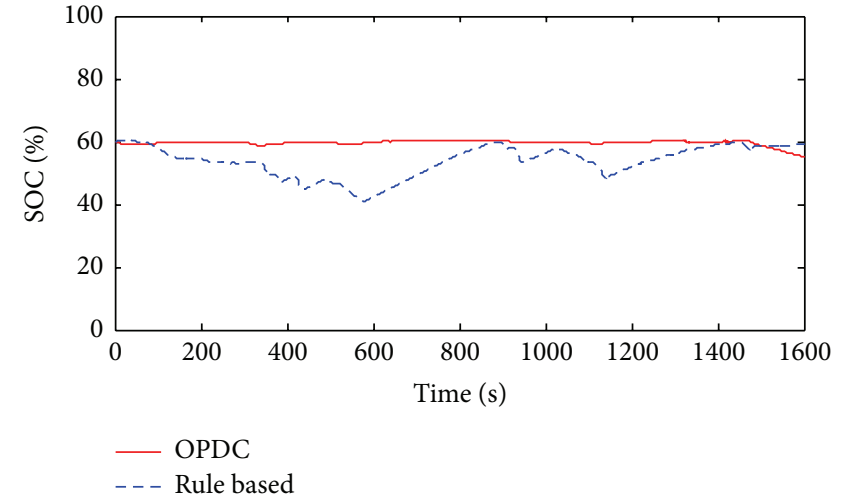

(a) SOC

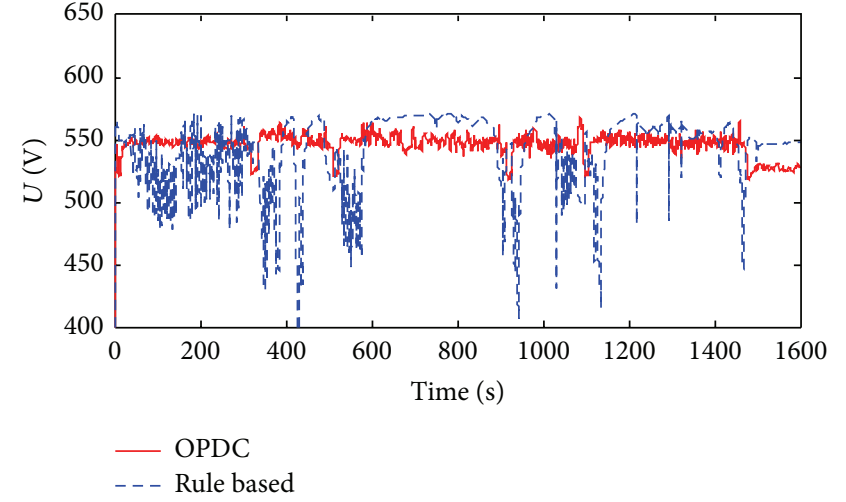

(b) DC bus voltage

FIGURE 16: States of the electrical system.

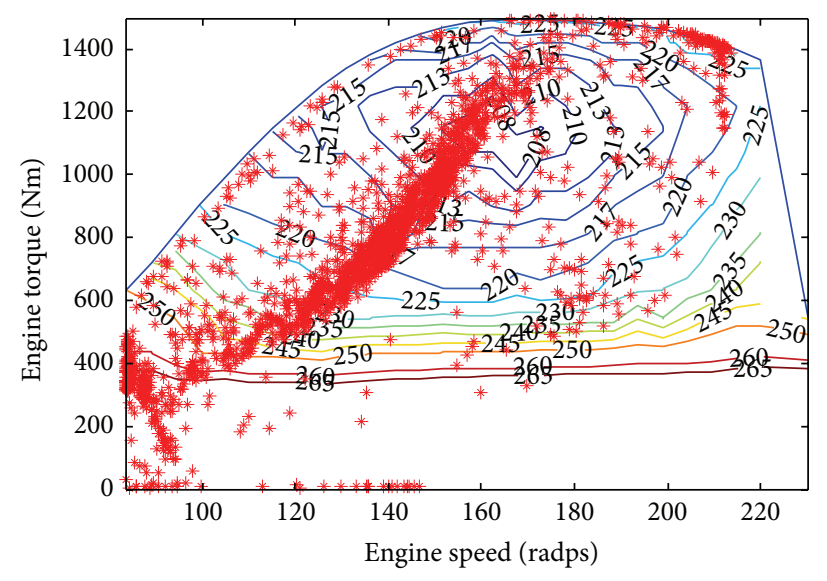

(a) OPDC

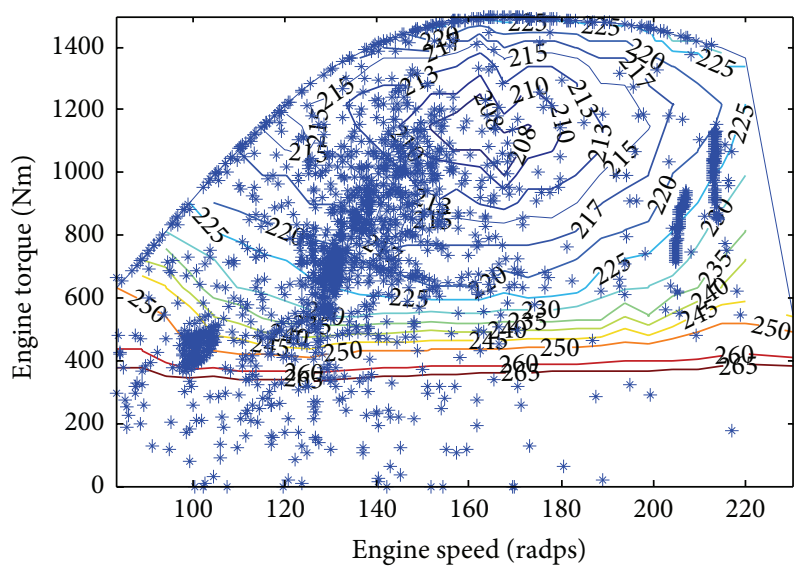

(b) Rule-based

FIGURE 17: Comparison of the engine's working points.

TABLE 2: Comparison analysis of the control effects.

\begin{tabular}{lccc}
\hline Cost function & Rule-based & OPDC & Improvement \\
\hline$J_{D} / \mathrm{kwh}$ & 22.71 & 16.15 & $28.9 \%$ \\
$J_{E} / \mathrm{kwh}$ & 3.6 & 1.89 & $47.5 \%$ \\
$J_{F} / \mathrm{L}$ & 13.45 & 10.88 & $19.1 \%$ \\
\hline
\end{tabular}

(region), that is, sacrificing the fuel economy to ensure the comprehensive performance.

The qualitative comparison of the two control approaches has been done in the past. Table 2 shows the quantitative comparison of the two control approaches.

In the OPDC approach, the index functions of drivability, current supply capacity, and fuel economy, $L_{D}, L_{E}$, and $L_{F}$, can be obtained through (21), (26), and (27). In the rule-based method, it is necessary to measure its control effects and make the comparison with the results of the OPDC approach.

It can be seen from Table 2 that compared with the rulebased approach, the drivability, current supply capacity, and fuel economy under the OPDC approach have been improved by $28.9 \%, 47.5 \%$, and $19.1 \%$, respectively. Thus, the OPDC control approach can obviously improve the comprehensive performance of the HEV system and, therefore, has a very good research value.

5.2. On-Line Test. The simulation has been carried out by using the MATLAB software, which verifies the effectiveness of the OPDC approach. Based on this simulation, the online test is implemented by utilizing the dSPACE platform to verify the real-time performance of the OPDC approach.

The dSPACE platform, which can realize seamless connection with the MATLAB/SIMULINK, has been widely used in the development and test process of control algorithms. The ControlDesk, a comprehensive test software based on the dSPACE platform, is mainly applied in the online control process of the rapid control prototype (RCP) and the hardware-in-loop simulation (HIL).

Firstly, based on the simulation model, the CAN communication interface of the control unit and the vehicle is established by using the RTI model store (see Figure 18). The control unit and the vehicle model send and receive the data through CAN1 and CAN2, respectively. The types of data 


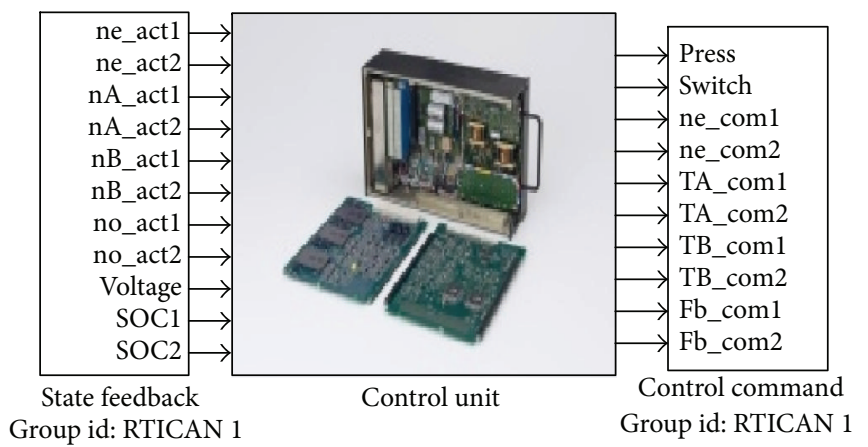

(a) Control unit

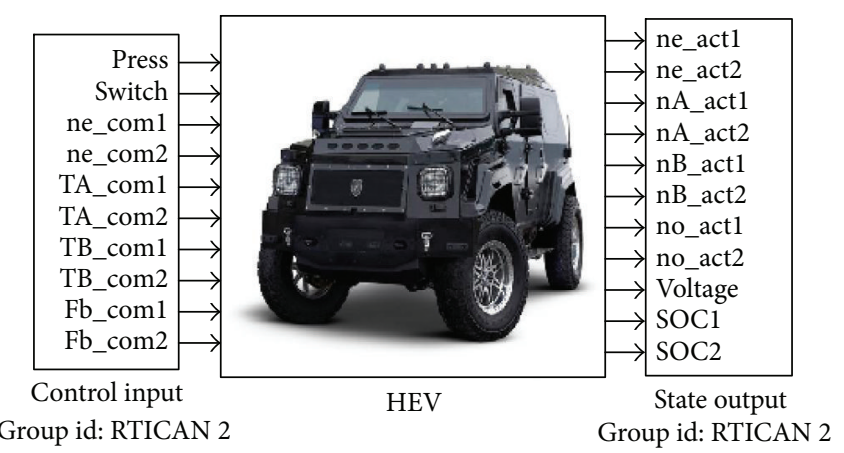

(b) Vehicle

FIGURE 18: Communication interface of the on-line test platform.

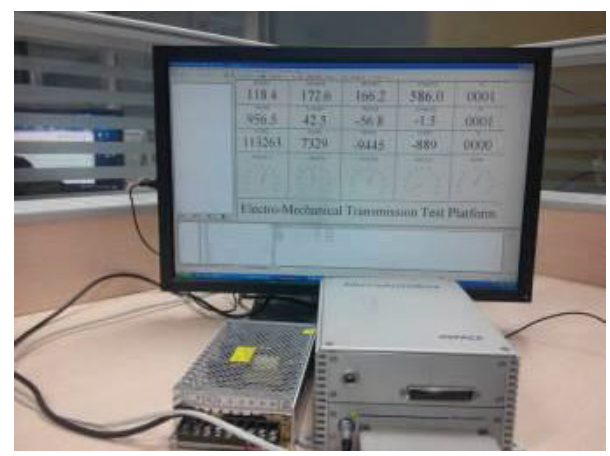

FIGURE 19: On-line test platform.

sent by CAN1 and received by CAN2 correspondingly match. They are the same with the types of data received by CAN1 and sent by CAN2.

Secondly, the models are transformed to $\mathrm{C}$ code using the MATLAB code-generation toolbox and downloaded to the two chips of the MicroAutoBox (hardware system of the dSPACE platform). The two chips, simulating the controller and vehicle, are connected with each other by the external CAN bus, through which they are able to realize real-time data communication.

Lastly, the experimental interface is established by using the ControlDesk, and the on-line test is conducted, as shown in Figure 19. The vehicle model receives the control variables through the CAN bus and sends the state information back to the CAN bus. By using the state information received from the CAN bus, the on-line optimization based on the OPDC algorithm is carried out, and the optimal control variables are sent back to the CAN bus. It, thus, can be seen that the controller and the vehicle model realize real-time communication through the CAN bus. Their operation condition is displayed on the ControlDesk experimental interface in real time, and the experimental data are automatically saved, so they can be used conveniently for the analysis.

The test results are basically the same with those of the dynamic simulation, which means that the OPDC control approach has a good real-time performance. The online test results can be analyzed based on Figure 19, and the experiment curves will not be given repeatedly. The dSPACE platform can be directly used in the real-time control process as a top-level controller, which can greatly save the developing time of the actual controller.

\section{Conclusion}

The existing control strategies of the HEVs mainly deal with energy management between the engine and the battery to realize the best fuel economy and emission performance. However, in the EMT system, the battery's power is far less than the engine's power, which means that energy management makes little sense. The dynamic control of the engine and the MGs is the main problem in the EMT system. As these components are coupled together by the coupling mechanism and the power unit and all of them have strong nonlinear characteristics, it is challenging to develop an optimal control method for the EMT system.

The multiobjective nonlinear dynamic optimization model of the HEV, equipped with a dual-mode EMT system, is derived based on the characteristics of the system, such as the performance requirements for drivability, fuel economy and current supply capacity, the nonlinear coupling constraints of the battery, the MGs, and the electric appliances. Based on the dynamic model of the system, a novel nonlinear optimal control approach is proposed in this paper, which consists of state observation, dynamic prediction, optimal decision, and feedback compensation. The state observation models of the engine's torque and the driving resistance are created based on the dynamic and coupling characteristics of the EMT system, and the optimal state estimation is conducted using the Kalman filtering algorithm. In the dynamic prediction part, the constraints are divided into single constraints, simple coupling constraints, and complex coupling constraints, and the hierarchical search mechanism is also adopted. A multiobjective hierarchical optimization algorithm, which transforms the multiobjective optimization problem to several single-objective ones within different hierarchies, is proposed in the optimal decision part, and the nonlinear programming algorithm based on feasible directions method is adopted to solve the nonlinear optimization problem. 
The optimization strategy and the feedback control are combined together in the feedback compensation part, so that the real-time performance and the effectiveness of the control algorithm are guaranteed.

The dynamic simulation platform of the HEV is established, and the simulation results verify the efficiency of the OPDC approach. Compared with the rule-based approach, the drivability, current supply capacity, and fuel economy are improved by $28.9 \%, 79.4 \%$, and $19.1 \%$, respectively, under the OPDC approach. Then, the on-line test is carried out based on the dSPACE platform, which verifies the real-time performance of the OPDC approach and lays the foundation for its real-time implementation.

\section{Conflict of Interests}

The authors declare that there is no conflict of interests regarding the publication of this paper.

\section{Acknowledgments}

This work was supported by the National Natural Science Foundation of China (Grant nos. 51005017, 51205020, and 51305026) and the Natural Science Program for New Century Excellent Talents in University (NCET-12-0043).

\section{References}

[1] F. R. Salmasi, "Control strategies for hybrid electric vehicles: evolution, classification, comparison, and future trends," IEEE Transactions on Vehicular Technology, vol. 56, no. 5, pp. 23932404, 2007.

[2] A. Panday and H. O. Bansal, "A review of optimal energy management strategies for hybrid electric vehicle," International Journal of Vehicular Technology, vol. 2014, Article ID 160510, 19 pages, 2014.

[3] R. Wang and S. M. Lukic, "Dynamic programming technique in hybrid electric vehicle optimization," in Proceedings of the IEEE International Electric Vehicle Conference (IEVC '12), IEEE, March 2012.

[4] L. V. Pérez, G. R. Bossio, D. Moitre, and G. O. García, "Optimization of power management in an hybrid electric vehicle using dynamic programming," Mathematics and Computers in Simulation, vol. 73, no. 1-4, pp. 244-254, 2006.

[5] Y. Zou, H. Shi-Jie, L. Dong-Ge, G. Wei, and X.-S. Hu, "Optimal energy control strategy design for a hybrid electric vehicle," Discrete Dynamics in Nature and Society, vol. 2013, Article ID 132064, 8 pages, 2013.

[6] A. P. Pourhashemi, S. M. M. A. Movahed, and M. S. Panahi, "Application of the fuel-optimal energy management in design study of a parallel hybrid electric vehicle," Journal of Fuels, vol. 2014, Article ID 417172, 12 pages, 2014.

[7] X. Fu, H. Wang, N. Cui, and C. Zhang, "Energy management strategy based on the driving cycle model for plugin hybrid electric vehicles," Abstract and Applied Analysis, vol. 2014, Article ID 341096, 6 pages, 2014.

[8] C. Musardo, G. Rizzoni, Y. Guezennec, and B. Staccia, "AECMS: an adaptive algorithm for hybrid electric vehicle energy management," European Journal of Control, vol. 11, no. 4-5, pp. 509-524, 2005.
[9] C. Vagg, C. J. Brace, S. Akehurst, and L. Ash, "Minimizing battery stress during hybrid electric vehicle control design," in Proceedings of the 9th IEEE Vehicle Power and Propulsion Conference (VPPC '13), University of Bath, 2013.

[10] B. Gu and G. Rizzoni, "An adaptive algorithm for hybrid electric vehicle energy management based on driving pattern recognition," in Proceedings of the ASME International Mechanical Engineering Congress and Exposition (IMECE '06), November 2006.

[11] Y. Zou, F. Sun, X. Hu, L. Guzzella, and H. Peng, "Combined optimal sizing and control for a hybrid tracked vehicle," Energies, vol. 5, no. 11, pp. 4697-4710, 2012.

[12] L. Johannesson and B. Egardt, "A novel algorithm for predictive control of parallel hybrid powertrains based on dynamic programming," Advances in Automotive Control, vol. 5, no. 1, pp. 343-350, 2007.

[13] P. Pisu and G. Rizzoni, "A supervisory control strategy for series hybrid electric vehicles with two energy storage systems," in Proceedings of the IEEE Vehicle Power and Propulsion Conference (VPPC '05), IEEE, September 2005.

[14] P. Tulpule, V. Marano, and G. Rizzoni, "Energy management for plug-in hybrid electric vehicles using equivalent consumption minimisation strategy," International Journal of Electric and Hybrid Vehicles, vol. 2, no. 4, pp. 329-350, 2010.

[15] M. Sivertsson, "Adaptive control using map-based ECMS for a PHEV," in Proceedings of the IFAC Workshop on Engine and Powertrain Control, Simulation and Modeling (E-COSM '12), pp. 357-362, October 2012.

[16] S. Overington and S. Rajakaruna, "Review of PHEV and HEV operation and control research for future direction," in Proceedings of the 3rd IEEE International Symposium on Power Electronics for Distributed Generation Systems (PEDG '12), pp. 385-392, IEEE, Aalborg, Denmark, June 2012.

[17] L. Johannesson, M. Åsbogård, and B. Egardt, "Assessing the potential of predictive control for hybrid vehicle powertrains using stochastic dynamic programming," IEEE Transactions on Intelligent Transportation Systems, vol. 8, no. 1, pp. 71-83, 2007.

[18] J. Tate, J. W. Grizzle, and H. Peng, "Shortest path stochastic control for hybrid electric vehicles," International Journal of Robust and Nonlinear Control, vol. 18, no. 14, pp. 1409-1429, 2008.

[19] Z. Lu, J. Song, H. Yuan, and L. Shen, "MPC-based torque distribution strategy for energy management of power-split hybrid electric vehicles," in Proceedings of the 32nd Chinese Control Conference (CCC '13), pp. 7650-7655, IEEE, July 2013.

[20] S. J. Qin and T. A. Badgwell, "A survey of industrial model predictive control technology," Control Engineering Practice, vol. 11, no. 7, pp. 733-764, 2003.

[21] T. S. Kim, C. Manzie, and R. Sharma, "Model predictive control of velocity and torque split in a parallel hybrid vehicle," in Proceedings of the IEEE International Conference on Systems, Man and Cybernetics (SMC '09), pp. 2014-2019, IEEE, San Antonio, Tex, USA, October 2009.

[22] H. Borhan, A. Vahidi, A. M. Phillips, M. L. Kuang, I. V. Kolmanovsky, and S. Di Cairano, "MPC-based energy management of a power-split hybrid electric vehicle," IEEE Transactions on Control Systems Technology, vol. 20, no. 3, pp. 593-603, 2012.

[23] M. Bichi, G. Ripaccioli, S. Di Cairano, D. Bernardini, A. Bemporad, and I. V. Kolmanovsky, "Stochastic model predictive control with driver behavior learning for improved powertrain control," in Proceedings of the 49th IEEE Conference on Decision 
and Control (CDC '10), pp. 6077-6082, IEEE, Atlanta, Ga, USA, December 2010.

[24] S. D. Cairano, D. Bernardini, A. Bemporad, and I. V. Kolmanovsky, "Stochastic MPC with learning for driver-predictive vehicle control and its application to HEV energy management," IEEE Transactions on Control Systems Technology, vol. 22, no. 3, pp. 1018-1031, 2014.

[25] R. C. B. Sampaio, A. C. Hernandes, V. D. V. M. Fernandes, M. Becker, and A. A. G. Siqueira, "A new control architecture for robust controllers in rear electric traction passenger HEVs," IEEE Transactions on Vehicular Technology, vol. 61, no. 8, pp. 3441-3453, 2012.

[26] A. Rajagopalan and G. Washington, "Intelligent control of hybrid electric vehicles using GPS information," SAE Technical Paper 2002-01-1936, SAE, 2002.

[27] C. Dextreit and I. V. Kolmanovsky, "Game theory controller for hybrid electric vehicles," IEEE Transactions on Control Systems Technology, vol. 22, no. 2, pp. 653-663, 2014.

[28] M. Zhang, Y. Yang, and C. C. Mi, "Analytical approach for the power management of blended-mode plug-in hybrid electric vehicles," IEEE Transactions on Vehicular Technology, vol. 61, no. 4, pp. 1554-1566, 2012.

[29] M. Song, J. Oh, S. Choi, Y. Kim, and H. Kim, "Optimal line pressure control for an automatic transmission-based parallel hybrid electric vehicle considering mode change and gear shift," Advances in Mechanical Engineering, vol. 6, Article ID 216098, 2014.

[30] S. C. Garnett and E. D. Stemler, "Electro-mechanical transmission,” US Patent no. 5,980,410, 1999.

[31] G. Welch and G. Bishop, "An introduction to the Kalman filter," 1995.

[32] M. Mastali, J. Vazquez-Arenas, R. Fraser, M. Fowler, S. Afshar, and M. Stevens, "Battery state of the charge estimation using Kalman filtering," Journal of Power Sources, vol. 239, pp. 294307, 2013.

[33] Z. Donghao, X. Changle, H. Lijin, and Z. Hailiang, "Multiobjective power flow hierarchic optimization for the ElectroMechanical Transmission of a heavy-duty vehicle," in Proceedings of the IEEE Transportation Electrification Conference and Expo, Asia-Pacific (ITEC Asia-Pacific '14), pp. 1-6, IEEE, Beijing, China, August 2014.

[34] Z. Xie, J. Li, and Z. Chen, Nonlinear Optimazation Theory and Methods, Higher Education Press, Beijing, China, 2012. 


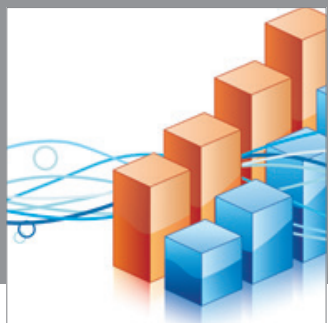

Advances in

Operations Research

mansans

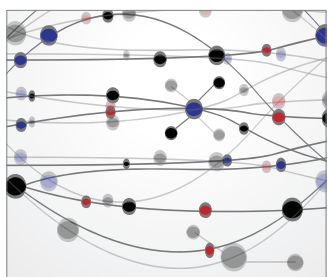

The Scientific World Journal
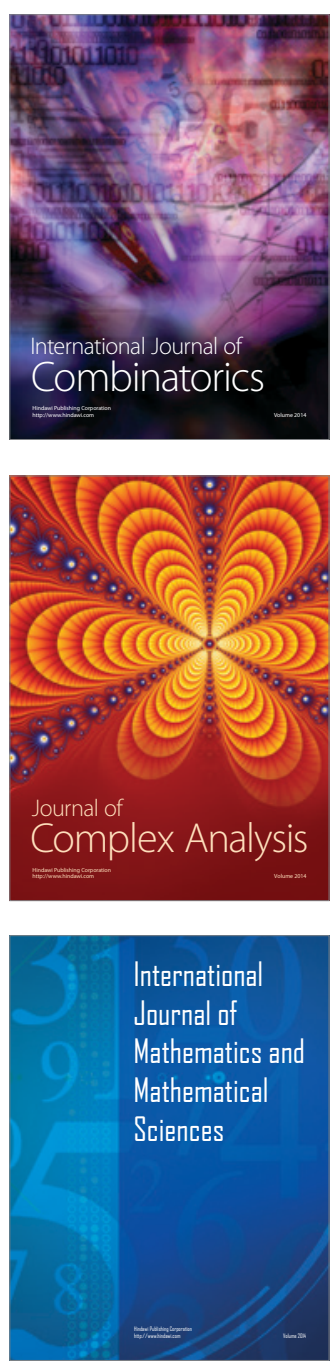
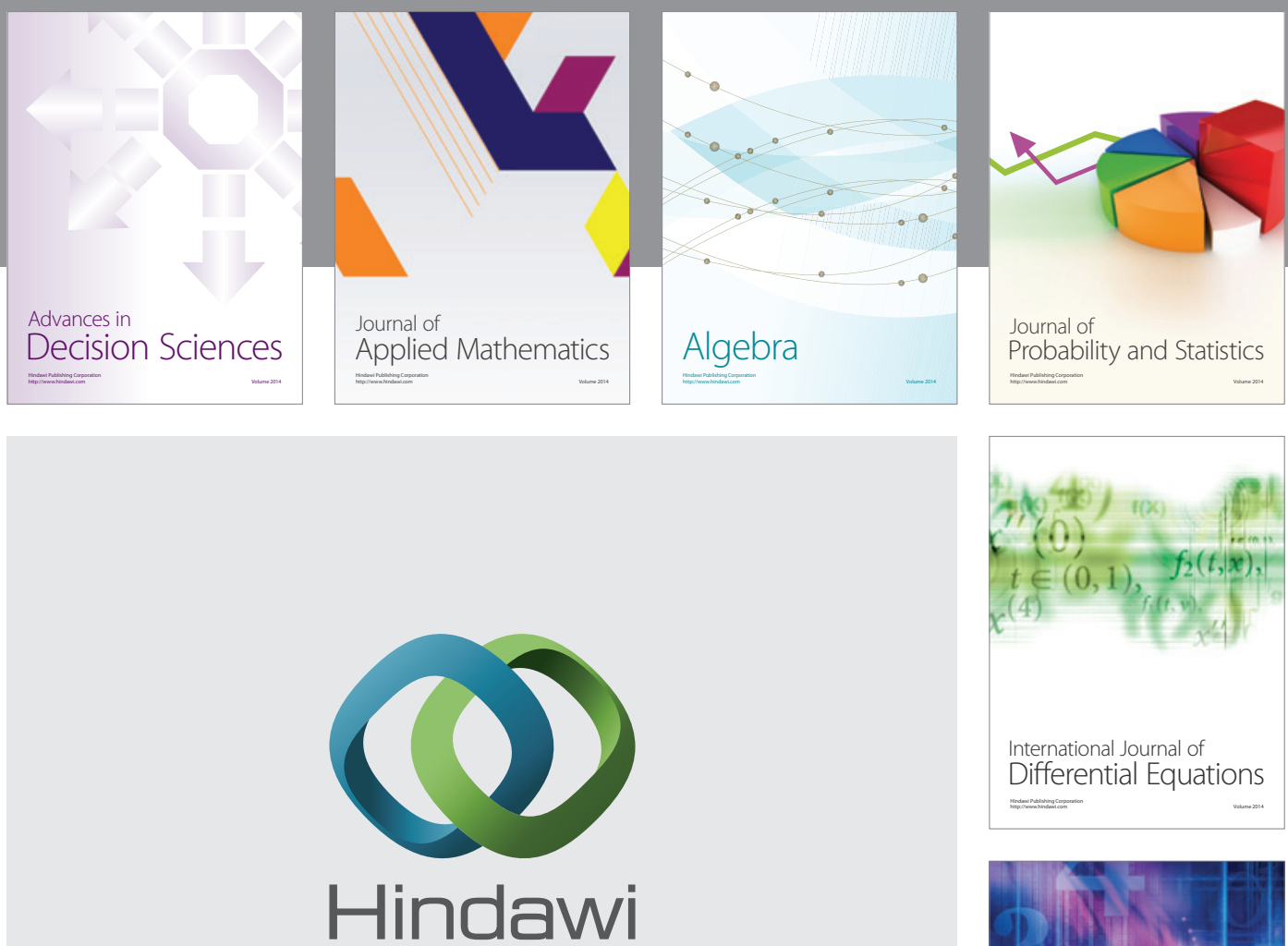

Submit your manuscripts at http://www.hindawi.com
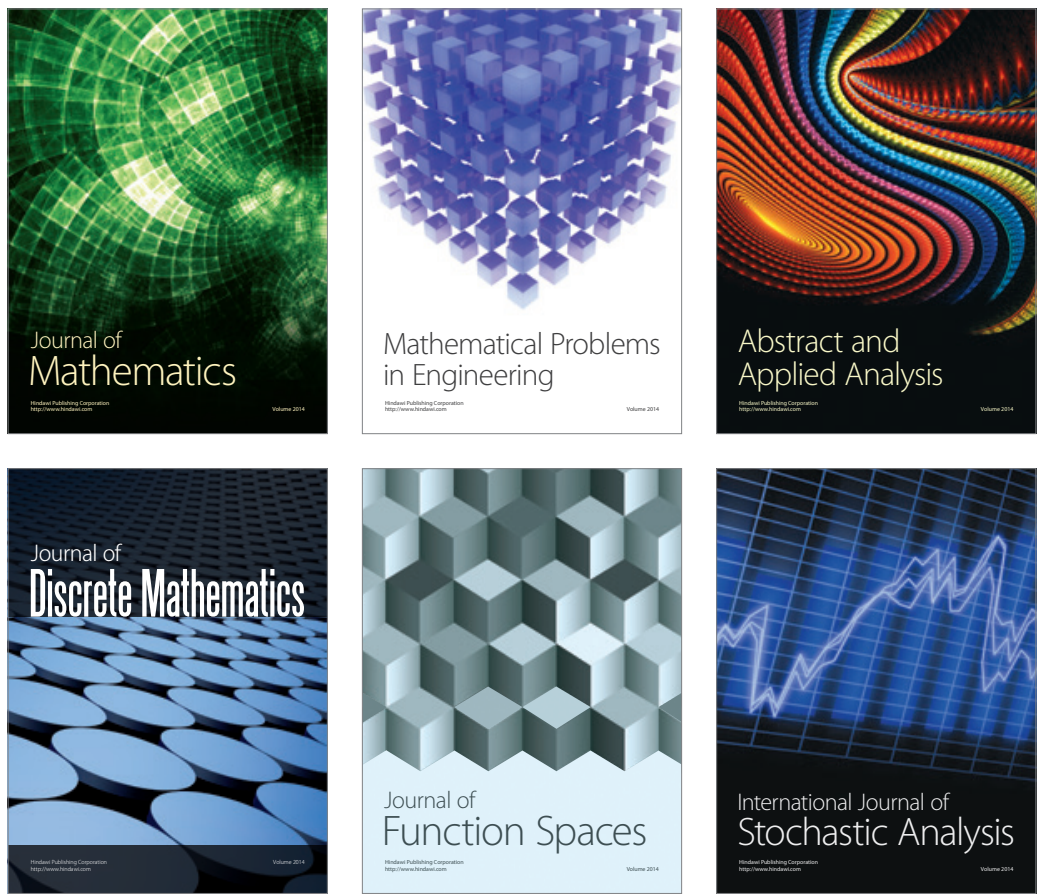

Journal of

Function Spaces

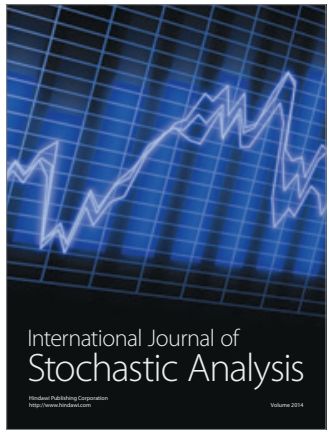

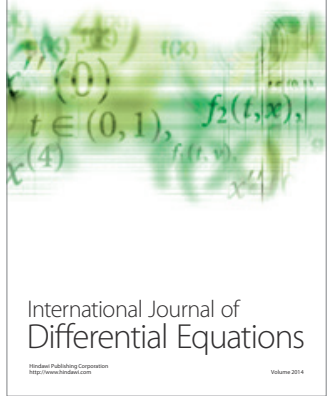
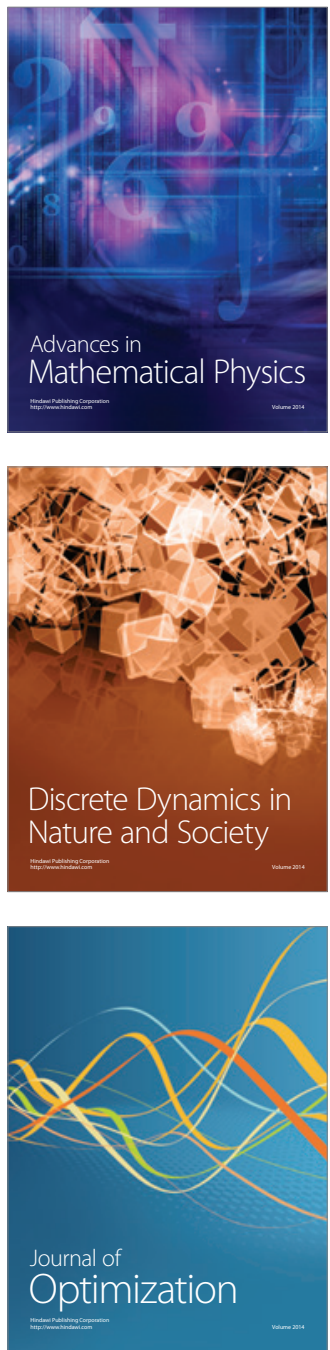